\title{
Review Article \\ Caveolin-1: An Oxidative Stress-Related Target for Cancer Prevention
}

\author{
Shengqi Wang, ${ }^{1,2,3}$ Neng Wang, ${ }^{1,2,4}$ Yifeng Zheng, ${ }^{1,2,3}$ Jin Zhang, ${ }^{2}$ Fengxue Zhang, ${ }^{2}$ and \\ Zhiyu Wang ${ }^{1,2,3}$ \\ ${ }^{1}$ Department of Mammary Disease, Discipline of Integrated Chinese and Western Medicine in Guangzhou University of Chinese \\ Medicine, The Second Affiliated Hospital of Guangzhou University of Chinese Medicine, Guangzhou, Guangdong, China \\ ${ }^{2}$ The Research Center for Integrative Medicine, Guangzhou University of Chinese Medicine, Guangzhou, Guangdong, China \\ ${ }^{3}$ Post-Doctoral Research Center, Guangzhou University of Chinese Medicine, Guangzhou, Guangdong, China \\ ${ }^{4}$ Department of Breast Oncology, Sun Yat-Sen University Cancer Center, State Key Laboratory of Oncology in South China, \\ Collaborative Innovation Center for Cancer Medicine, Guangzhou, Guangdong, China
}

Correspondence should be addressed to Zhiyu Wang; wangzhiyu976@126.com

Received 6 November 2016; Revised 23 January 2017; Accepted 7 March 2017; Published 4 May 2017

Academic Editor: Ilaria Peluso

Copyright (C) 2017 Shengqi Wang et al. This is an open access article distributed under the Creative Commons Attribution License, which permits unrestricted use, distribution, and reproduction in any medium, provided the original work is properly cited.

\begin{abstract}
Aberrant oxidative metabolism is one of the hallmarks of cancer. Reactive species overproduction could promote carcinogenesis via inducing genetic mutations and activating oncogenic pathways, and thus, antioxidant therapy was considered as an important strategy for cancer prevention and treatment. Caveolin-1 (Cav-1), a constituent protein of caveolae, has been shown to mediate tumorigenesis and progression through oxidative stress modulation recently. Reactive species could modulate the expression, degradation, posttranslational modifications, and membrane trafficking of Cav-1, while Cav-1-targeted treatments could scavenge the reactive species. More importantly, emerging evidences have indicated that multiple antioxidants could exert antitumor activities in cancer cells and protective activities in normal cells by modulating the Cav-1 pathway. Altogether, these findings indicate that Cav-1 may be a promising oxidative stress-related target for cancer antioxidant prevention. Elucidating the underlying interaction mechanisms between oxidative stress and Cav-1 is helpful for enhancing the preventive effects of antioxidants on cancer, for improving clinical outcomes of antioxidant-related therapeutics in cancer patients, and for developing Cav-1 targeted drugs. Herein, we summarize the available evidence of the roles of Cav-1 and oxidative stress in tumorigenesis and development and shed novel light on designing strategies for cancer prevention or treatment by utilizing the interaction mode between Cav-1 and oxidative stress.
\end{abstract}

\section{Introduction}

The cumulative production of reactive species, particularly reactive oxygen species (ROS) and reactive nitrogen species (RNS), through either endogenous or exogenous insults is termed oxidative stress [1]. Oxidative stress has been reported to be implicated in the etiology and progression of multiple human diseases, such as neurodegenerative disease, inflammatory disease, cardiovascular disease, allergies, immune system dysfunctions, diabetes, and aging as well as cancer [1]. Reactive species play an important role in cancer etiology and progression and are being progressively elucidated [2-4]. Physiological levels of reactive species are crucial for ensuring cell survival. However, overproduction of reactive species is detrimental to cells and could induce tumorigenesis by oxidizing specific intracellular moieties, resulting in genetic mutations and activations of ontogenetic pathways that stimulate proliferation and neoplastic transformation [2-5]. Cancer cells are usually submitted to higher levels of reactive species as a result of aberrations in oxidative metabolism (e.g., impaired mitochondrial oxidative phosphorylation [6] and increased aerobic glycolysis [7]) and signaling pathways (e.g., Ras [8] and AKT [9] activation), which further stimulate the malignant phenotypes of death evasion, angiogenesis, invasiveness, and metastasis [10]. Therefore, antioxidant therapies or dietary 
supplementations of antioxidants have gained considerable interests in cancer prevention [11-15]. In addition, cancer patients widely take antioxidant supplements in order to lower oxidative damages of chemotherapy and radiation therapies to normal tissues [16-19]. It is estimated that approximately $45-80 \%$ of breast cancer patients use antioxidant supplements after diagnosis or during breast cancer treatment [17]. Common antioxidants used by cancer patients include glutathione (GSH), $\beta$-carotene, vitamin A (retinoic acid), vitamin C (ascorbic acid), vitamin $\mathrm{E}$, and selenium $[11,13,14]$.

Caveolae are 50 to $100 \mathrm{~nm}$ flask-shaped invaginations of the plasma membrane which have important roles in membrane trafficking (endocytosis and transcytosis), maintaining membrane lipid composition and cell signal transduction [20]. Cav-1, the major structural protein of caveolae, functions as scaffolding protein [21] regulating multiple physiological processes including caveola biogenesis, vesicular transport, cholesterol homeostasis, and signal transduction [22]. Recently, Cav-1 has been demonstrated to be closely involved in tumorigenesis and development, affecting the proliferation $[23,24]$, survival $[25,26]$, apoptosis $[27,28]$, migration [29, 30], invasion [24, 31], metastasis [32, 33], autophagy [34, 35], transformation [36], anoikis [37, 38], and chemotherapy resistance $[39,40]$ processes of cancer cells. Cav-1 is generally regarded as a tumor suppressor, and studies have implicated loss of Cav-1 in the pathogenesis and progression of multiple human cancers [41-44]. For example, loss of Cav-1 in cancer-associated fibroblasts (CAFs) could result in an activated tumor microenvironment, thereby driving early tumor recurrence, metastasis, and poor clinical outcomes in breast cancer [45]. In contrast, overexpression of Cav-1 in CAFs predicted a good outcome [45]. On the other hand, Cav-1 is also regarded as an oncoprotein in some kinds of malignancies. It was reported that the expression of Cav-1 was related to poor prognosis in lung pleomorphic carcinoma [46] and prostate carcinoma $[47,48]$. In addition, Liu's group demonstrated that Cav-1 exhibited a stage-dependent, functional fluctuation during the progression of pancreatic cancer [49].

Recently, increasing evidences have showed that the oxidative stress processes in cancer cells were closely associated with Cav-1 [50-52]. Cav-1 is suggested to be a potential oxidative stress-related target during oxidative stressinduced cancer initiation and development. Reactive species overproduced in oxidative stress processes could modulate the expression [53], degradation [54], posttranslational modifications [55-57], and membrane trafficking [58] of Cav-1. Meanwhile, Cav-1 also has a feedback regulation effect on oxidative stress status in tumor microenvironment [50, 52]. For example, Cav-1 could attenuate hydrogen peroxideinduced oxidative damages to lung carcinoma cells [52]. More importantly, emerging evidences have indicated that multiple antioxidants could exert antitumor activities in cancer cells and protective activities in normal cells by modulating the Cav-1 pathway [59-61]. For example, treatment with antioxidants (such as quercetin, $\mathrm{N}$-acetyl-cysteine, and metformin) was reported to reverse Cav-1 loss-induced phenotypes, such as mitochondrial dysfunction, oxidative stress, and aerobic glycolysis in CAFs [60]. Altogether, these findings indicate that Cav-1 may be a promising oxidative stress-related target for cancer antioxidant prevention. Considering the critical role of oxidative stress in mediating the therapeutic efficacies of radiotherapy and chemotherapy, understanding the action mechanisms of Cav-1 during oxidative stress processes is important for improving the clinical outcomes and for developing novel diagnostic tools and drugs for cancer antioxidant prevention. Herein, we summarize the current evidence of the roles of Cav-1 and oxidative stress, as well as their interaction in tumorigenesis and progression.

\section{Structure, Localization, and Biofunctions of Cav-1}

Originally named to describe the cave-like invaginations on the cell surface, caveolae are 50-100 nm membrane microdomains which represent a subcompartment of the plasma membrane and are enriched in glycosphingolipids, cholesterol, and lipid-anchored membrane proteins [22, 62]. Caveolins are caveolae-resident structural and scaffolding proteins, which are critical for the formation of caveolae and their interaction with signaling entities [22]. Each caveola contains approximately 100 to 200 caveolin molecules. There are three isoforms of caveolins (Cav-1, Cav-2, and Cav-3), while Cav-1 is the principal component of caveolae. Cav-1 (21-24 KDa) is an integral membrane protein with an unusual hairpin-like conformation (Figure 1(a)). The N- and C-terminal cytoplasmic tails of Cav1 are separated by a hydrophobic segment that does not cross the membrane [63-65]. Both the C- and N-termini face the cytoplasm [66]. Cav-1 oligomerizes through amino acids $1-101$. This oligomerization region contains the scaffolding domain (juxtamembrane 82-101 amino acids). Cav-1 has been identified primarily as two isoforms, Cav- $1 \alpha$ and Cav-1 $\beta$, which differs in their $\mathrm{N}$-termini and derives from alternative translation initiation sites [67]. The presence of Cav-1 has been verified in most cell types by biochemical and morphological experiments, including epithelial cells, endothelial cells, fibroblasts, smooth muscle cells, and adipocytes [22, 68, 69]. Although Cav-1 is expressed ubiquitously, the levels of its expression vary among different tissues [22]. Cellular organelles where Cav-1 is enriched include the mitochondrion, the nucleus, the Golgi complex, and the endoplasmic reticulum [21, 22].

As shown in Figure 1(b), Cav-1 is involved in the regulation of multiple physiological processes, including caveola biogenesis, vesicular transport, cholesterol homeostasis, lipid transport, mitochondrial function, and signal transduction [22]. Cav-1 is a molecular hub. It could integrate transduction and negatively regulate a variety of signaling molecules, which include mitogen-activated protein kinases (MAPKs), epidermal growth factor receptor (EGFR), Neu, Ras family GTPases, transforming growth factor- $\beta$ (TGF- $\beta$ ), G-protein-coupled receptors (GPCRs), Src family kinases, endothelial nitric oxide synthase (eNOS), adenylyl cyclases, protein kinase A (PKA), and AKT [22, 70-74]. The caveolin-signaling hypothesis proposes that the interactions between Cav-1 and these 


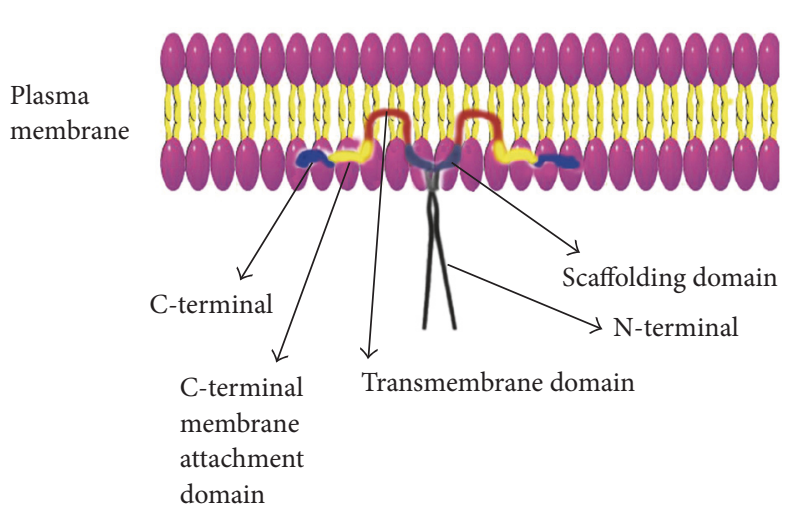

(a)

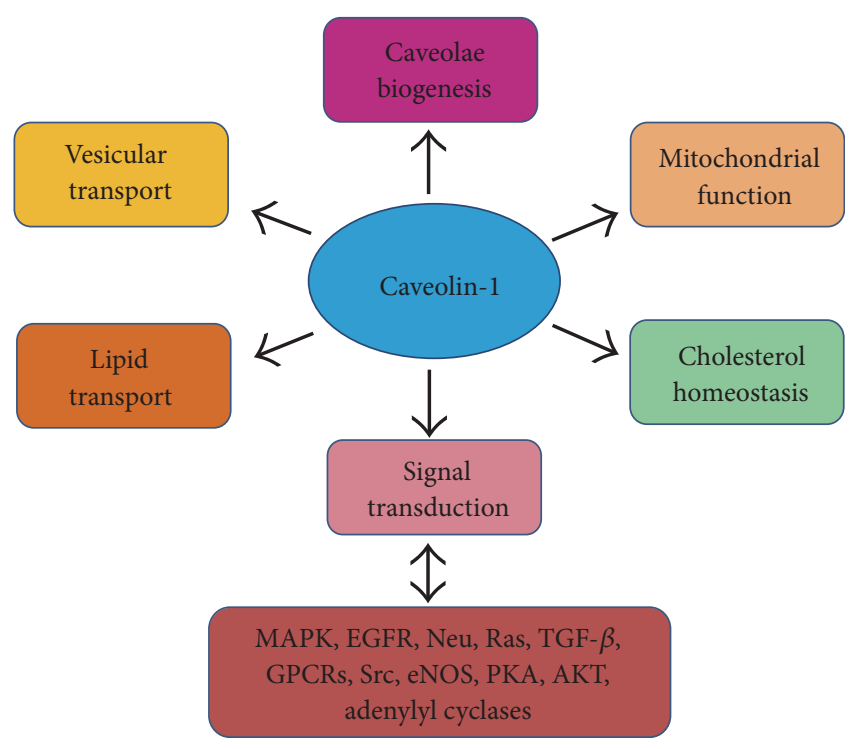

(b)

FIgURE 1: Structure and biofunctions of Cav-1. (a) The structure of Cav-1. The C-terminal, the C-terminal membrane attachment domain, and the transmembrane domain of Cav-1 are highlighted in blue, yellow, and red, respectively. The Cav-1-scaffolding domain (CSD) is responsible for Cav-1 interaction with other molecules. (b) Cav-1 biofunctions. Cav-1 is involved in the regulation of multiple physiological processes including caveola biogenesis, vesicular transport, cholesterol homeostasis, lipid transport, mitochondrial function, and signal transduction.

molecules are through the Cav-1-scaffolding domain (CSD) in Cav-1 molecule [72]. The Cav-1-scaffolding domain is also necessary for direct binding to cholesterol which participates in cholesterol transport and raft organization [75-77].

\section{Emerging Role of Cav-1 in Cancer Prevention}

As stated above, Cav-1 is a molecular hub-integrating transduction of multiple cellular molecules which are closely connected with the biological behaviors of cancer cells [78]. In addition, Cav-1 participates in multiple protein/protein and protein/lipid interactions which are critical for cell survival [22]. Undoubtedly, dysfunctional Cav-1 plays an important role in tumorigenesis and progression [79]. Human CAV1 gene is localized to a suspected tumor suppressor locus (7q31.1) [53], which is a fragile genomic region and often deleted in cancers, suggesting that Cav-1 is possibly a tumor suppressor $[80,81]$. The caveolin-signaling hypothesis proposes that Cav-1 could directly interact with multiple cancer-related signaling molecules including EGFR, Neu, TGF- $\beta$, Src, and AKT via the scaffolding domain and negatively modulate their aberrant activations. This hypothesis may further explain the role of Cav-1 as a tumor suppressor $[22,70]$. Recent studies have implicated Cav-1 loss in the pathogenesis of various types of human malignancies [82-94] (Table 1). In breast cancer, loss of stromal Cav-1 was identified as a predictive biomarker of early tumor recurrence, lymph node metastasis, and tamoxifenresistance as well as decreased survival in human breast cancer patients $[42,43,95,96]$, suggesting that Cav-1 functions as a tumor suppressor in breast cancer. Similar work confirmed that stromal Cav-1 loss was specifically associated with early ductal carcinoma in situ (DCIS) progression to invasive breast cancer, with shorter time to recurrence and higher recurrence rate [97]. In addition, Cav-1 is also negatively associated with breast cancer cells' transformation. Xie's group reported that Cav-1 expression was significantly attenuated in 3-phosphoinositidedependent protein kinase-1- (PDK1-) mediated transformation of mammary epithelial cells [36]. Importantly, the prognostic value of stromal Cav-1 loss in breast cancers has now been independently validated [98-101] and has been extended to multiple types of human cancers, such as colorectal cancer [44], advanced prostate cancer [102], metastatic melanoma [41], gastric cancer [103], and osteosarcoma [104]. In colorectal cancer, it has been reported that stromal Cav-1 loss predicted poor survival [44]. In advanced prostate cancer, an absence of stromal Cav-1 was associated with metastatic disease and epithelial AKT activation [102]. In metastatic melanoma, stromal Cav-1 loss predicted poor survival of malignant melanoma patients [41]. In gastric cancer, epithelial Cav-1 loss could promote malignant progression of gastric cancer, and Cav-1 loss in CAFs heralded worse outcome of gastric cancer patient, suggesting Cav-1 level in CAFs may be a candidate therapeutic target and a useful prognostic marker of gastric cancer [103]. In osteosarcoma, Cav-1 downregulation could unleash c-Src and Met signaling, enabling osteosarcoma cells to invade neighboring tissues [104]. Conversely, overexpression of Cav-1 in osteosarcoma cell lines brought reduced malignancy with inhibited anchorage-independent growth, migration, and invasion. In addition, Cav-1 has also been reported to be closely connected with the multidrug resistance (MDR) of cancer cells [105-108]. For example, it was reported that Cav-1 could sensitize cancer cells to apoptosis in 
TABLE 1: Caveolin-1 acts as a cancer suppressor in multiple malignancies.

\begin{tabular}{|c|c|c|c|}
\hline Cancer type & Biofunction & Mechanisms & Reference number \\
\hline Pancreatic cancer & Cancer suppressor & $\begin{array}{l}\text { Inhibiting the EGFR, c-Raf, Mek, and Erk pathways; } \\
\text { attenuating the expression of MMPs; inducing apoptosis; } \\
\text { and inducing cell cycle arrest in the G0/G1 phase }\end{array}$ & {$[30,90,91]$} \\
\hline Breast cancer & Cancer suppressor & $\begin{array}{l}\text { Inducing apoptosis, inducing cell cycle arrest in the G2/M phase, } \\
\text { suppressing glycolysis, downregulating the expression of growth } \\
\text { factors, inhibiting lysosomal function, inhibiting autophagy, } \\
\text { activating the } p 53 \text { pathway, inhibiting the AMPK pathway, } \\
\text { and inhibiting the } \mathrm{Ca}^{2+} \text {-activated potassium channels }\end{array}$ & $\begin{array}{l}{[32,34-36,40,42} \\
43,95,96,98-101]\end{array}$ \\
\hline Colon cancer & Cancer suppressor & $\begin{array}{l}\text { Inhibiting MMP-4 expression, facilitating cyclooxygenase-2, } \\
\text { and EGFR degradation }\end{array}$ & {$[33,44,92,93]$} \\
\hline Melanoma & Cancer suppressor & Suppressing the integrin/Src/FAK pathway & {$[41,94]$} \\
\hline Leukemia cancer & Cancer suppressor & $\begin{array}{l}\text { Inducing apoptosis, inducing the cell cycle arrest in the G1 phase, } \\
\text { suppressing the PI3K/AKT/mTOR pathway, suppressing } \\
\text { the VEGF redox signal transduction cascades }\end{array}$ & {$[82,84]$} \\
\hline Gastric Cancer & Cancer suppressor & $\begin{array}{l}\text { Inducing the expression of E-cadherin, inhibiting fibroblast } \\
\text { activation, suppressing the Ras/ MAPK pathway }\end{array}$ & {$[85-87,103]$} \\
\hline Rhabdomyosarcoma & Cancer suppressor & $\begin{array}{l}\text { Suppressing the MAPK pathway, promoting } \\
\text { muscular differentiation }\end{array}$ & [89] \\
\hline Advanced prostate cancer & Cancer suppressor & Suppressing epithelial Akt activation & {$[102]$} \\
\hline Osteosarcoma & Cancer suppressor & Inhibiting c-Src activity and the Met pathway & {$[104]$} \\
\hline
\end{tabular}

response to death stimuli, and a decrease of Cav-1 expression level was proved to contribute to chemotherapeutic cisplatin and carboplatin resistance [109].

Multiple clinical studies also verified the critical role of Cav-1 for cancer prevention. Bertino et al. observed that higher Cav-1 levels in tumor-associated stroma were significantly correlated with improved partial response rates $(P=0.036)$ and overall survival $(\mathrm{OS})(P=0.008)$ in advanced NSCLC patients, who treated with nanoparticle albuminbound (nab) paclitaxel plus with carboplatin [110]. Furthermore, in a large cohort of 178 patients with colorectal cancer, Zhao et al. observed that the loss of stromal Cav-1 expression was associated with shorter disease-free survival $(P=0.000)$ and shorter OS $(P=0.000)$. This study further showed that the expression level of stromal Cav-1 was closely associated with histological type $(P=0.022)$, pathologic tumor-nodemetastasis stage $(P=0.047)$, pathologic $\mathrm{N}$ stage $(P=0.035)$, and recurrence $(P=0.000)$ of colorectal cancer [44]. In a larger cohort of 724 patients with prostate cancer, Ayala and colleagues observed significantly decreased levels of stromal Cav-1 in concordance with increased Gleason score $(P=0.012)$ and reduced relapse-free survival $(P=0.009)$ [111]. Moreover, Jia et al. analyzed a total of 110 patients with esophageal squamous cell carcinoma and found that downregulation of stromal Cav-1 expression was associated with shorter disease-free survival $(P<0.001)$ and OS $(P<0.001)$, accompanied with more lymph node metastases $(P=0.020)$ and more local regional recurrences $(P=0.002)$ [112]. In addition, Yang et al. reported that increased Cav-1 expression was associated with prolonged overall survival rate in hepatocellular carcinoma $(P=0.021)$ [113]. Similar findings were also confirmed on breast cancer [99] and gastric cancer patients [114]. In particular, our analysis also revealed a close link between Cav-1 and lung cancer. Km-Plot analysis shows that lung cancer patients with $\mathrm{Cav}-1^{\text {high }}$ expression exhibit a better (OS) compared to those patients with Cav-1 ${ }^{\text {low }}$ expression (Figure 2(a)). Meanwhile, high Cav-1 expression also indicates a better OS for lung adenocarcinoma but not for squamous cell lung carcinoma (Figures 2(b) and 2(c)). What is more, in both lung cancer patients with smoke history or no, Cav-1 exhibits a positive correlation with better OS, indicating that Cav-1-targeting strategy might be effective for lung cancer patients independent of the factor of smoke (Figures 2(d) and 2(e)). Interestingly, Cav-1 $1^{\text {high }}$ expression also indicates an improved progression-free survival (PFS) and postprogression survival (PPS), further indicating the tumor suppressive role of Cav-1 in lung cancer (Figures 2(f) and 2(g)). In sum, accumulating evidence has indicated that Cav-1 might act as a tumor suppressor in multiple malignant tumors.

Interestingly, Cav-1 also acts as an oncoprotein depending on the tumor type and/or tumor stage. Particularly, Cav-1 seems to act as a tumor suppressor at early stages of cancer progression but as an oncoprotein in advanced-stage cancer [115]. For example, Cav-1 was found upregulated in multidrug-resistant colon cancer cells, adriamycin-resistant breast cancer cells, and taxol- and gemcitabine-resistant lung cancer cells $[108,116,117]$. Additionally, it was reported that CAV1 silencing could sensitize breast cancer stem cells (CSCs) by limiting their self-renewal ability but promoting the differentiation process [118]. The conflict roles of Cav-1 in tumor progression may be partly explained by the observation that Cav-1 has several peptide domains with opposing functions [83]. Tyrosine phosphorylation, serine phosphorylation, and dominant-negative point mutations in these domains serve to functionally inactivate the tumor suppressor function of Cav-1 [83]. Meanwhile, we previously 


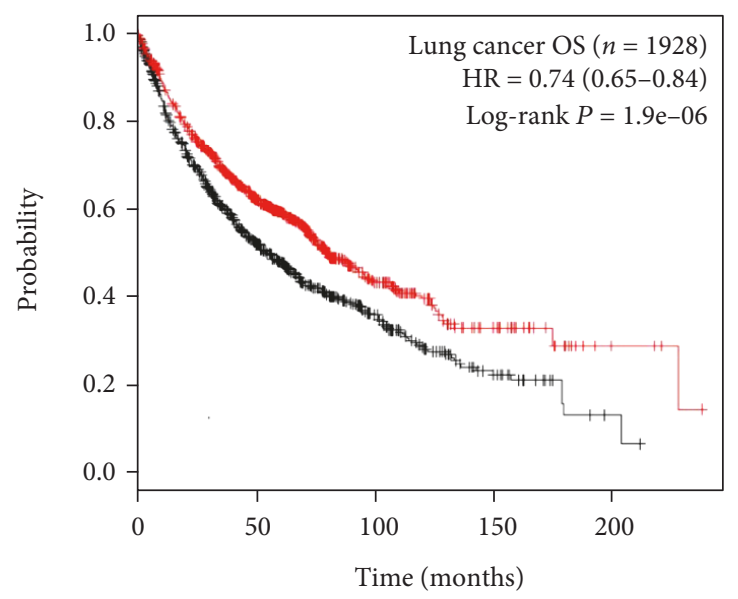

- Low expression

High expression

(a)

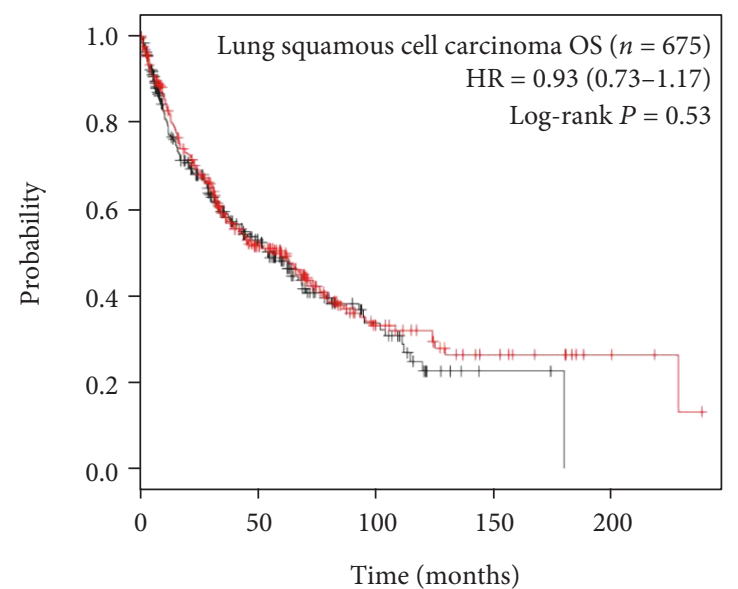

_ Low expression

High expression

(c)

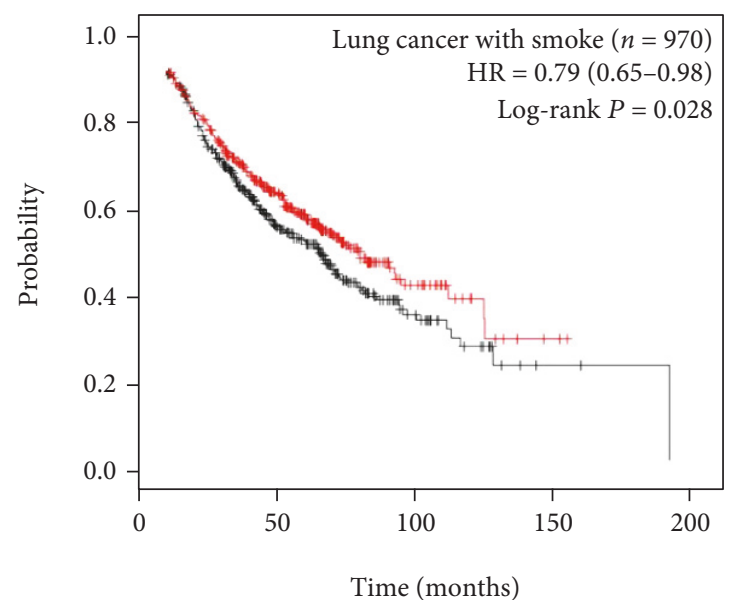

Time (months)

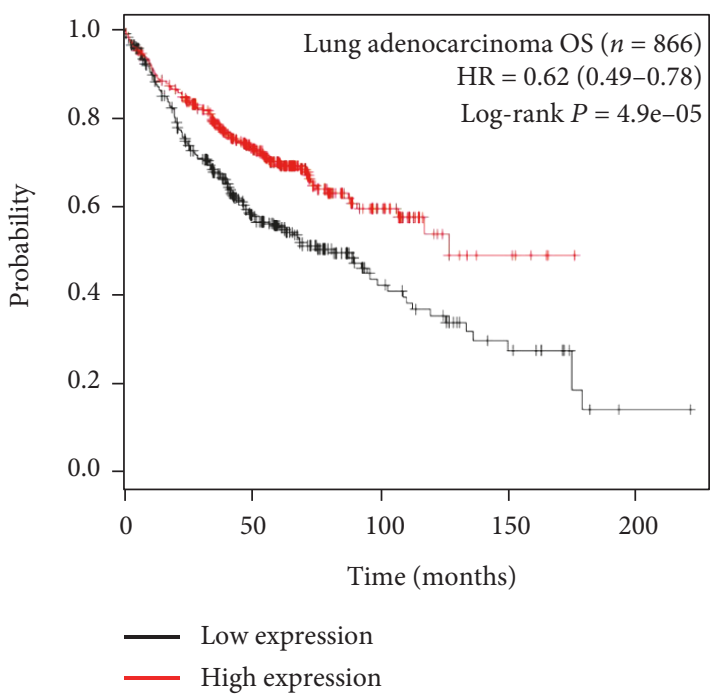

(b)

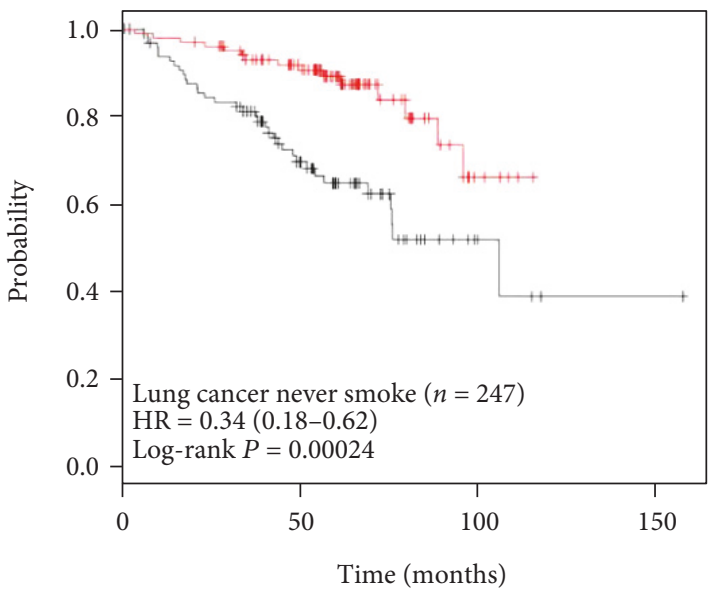

- Low expression High expression

(d)

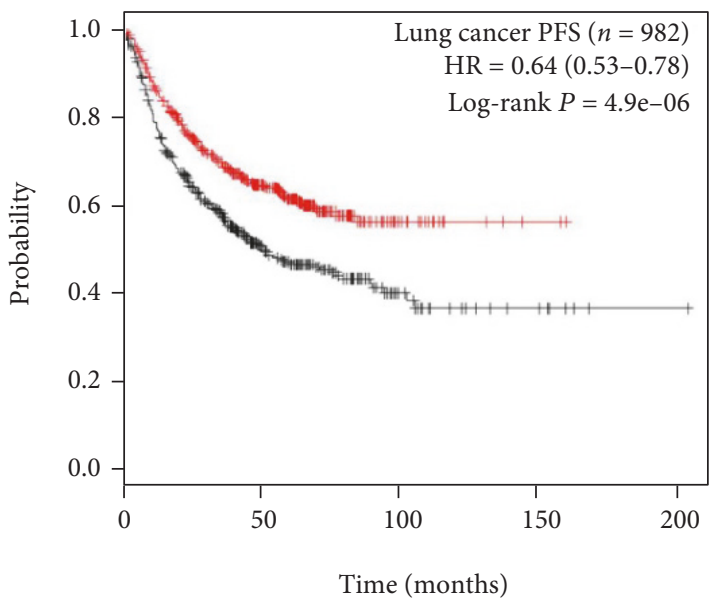

Low expression

High expression

(e)

(f)

Figure 2: Continued. 


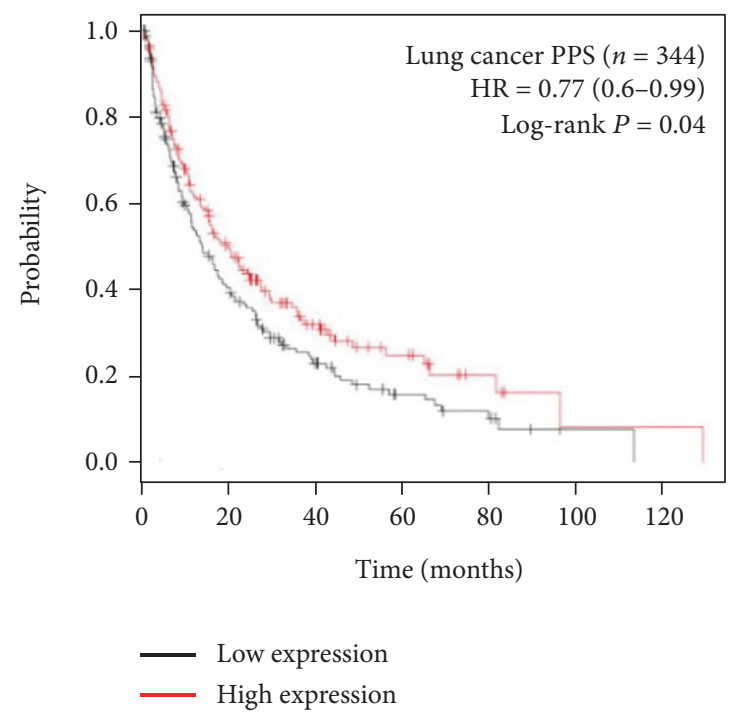

(g)

Figure 2: Tumor suppressive role of Cav-1 in lung cancer by Km-Plot analysis. (a) Cav-1 predicts an improved OS among lung cancer patients; (b-c) Cav-1 ${ }^{\text {high }}$ expression is closely correlated with improved OS in lung adenocarcinoma but not in squamous carcinoma; (d-e) the clinical significance of Cav-1 in predicting OS is independent of smoke history; (f-g) besides OS, Cav-1 ${ }^{\text {high }}$ expression is also significantly correlated with improved PFS and PPS.

proposed a Cav-1 fluctuation model during cancer development [119]. It is suggested that Cav-1 might act as a kind of stress signal, which protects cells from hazardous damage, but its loss may make cells more sensitive to oncogenic events. However, when cancer progresses into the advanced stage or are treated with cytotoxic agents, the expression of Cav-1 would be upregulated to protect cancer cells escaping from death by speeding aerobic glycolysis, increasing stem cell population or overexpressing $\mathrm{ABC}$ transporters. Therefore, it is easy to understand current confusing evidence of Cav-1 in cancer development, and Cav-1 resurrection strategy is promising for preventing normal cells from malignant transformation.

\section{Critical Role of Oxidative Stress Signaling in Mediating Cav-1 Anticarcinogenic Activities}

Although physiological levels of reactive species function as important signaling of certain subcellular events such as enzymatic activity [5], gene expression [120], and protein synthesis [121], elevated levels of reactive species could initiate multiple toxic oxidative reactions including initiation of lipid peroxidation, direct inhibition of membrane sodium/ potassium ATPase activity and mitochondrial respiratory chain enzymes, inactivation of glyceraldehyde-3-phosphate dehydrogenase, and membrane sodium channels [2-4]. All these toxicities are reported to play a role in carcinogenesis. In addition, elevated levels of reactive species could alter and damage many intracellular molecules, including nucleic acids, proteins, lipids, and polysaccharides [122], and therefore initiating a series of pathological processes and diseases. For example, reactive species could cause nicks in DNA, as well as malfunctions in the DNA repair mechanism. DNA oxidation induced by these reactive species generates 8 - hydroxy-2'-deoxyguanosine, a product that is able to generate mutations in DNA and enhances carcinogenesis [123]. Furthermore, cancer cells usually exhibit increased levels of reactive species [124], which were found to facilitate cancer growth through sustained proliferation, apoptosis resistance, death evasion, angiogenesis, invasiveness, metastasis, and hypoxia-inducible factor 1 (HIF-1) activation [125-129]. Therefore, eliminating elevated oxidative stress is considered as an important strategy for cancer prevention.

Recent studies also revealed the critical role of oxidative stress in Cav-1 loss-mediated carcinogenic process. CAV1 silencing in fibroblasts promoted ROS production, despaired mitochondrial activity, and DNA damage [50]. Similarly, treatment of fibroblasts with pro-oxidant buthionine sulfoxide caused the dose-dependent downregulation of Cav-1 [130]. In a coculture system, $C A V 1^{-1-}$ fibroblasts were validated to promote breast cancer growth via driving aerobic glycolysis. Remarkably, the tumor-promoting effects of $C A V 1^{-/-}$fibroblasts were reverted by recombinant overexpression of superoxide dismutase 2 (SOD2), thus implying that oxidative stress is critical for facilitating Cav-1 lossinduced carcinogenesis [131]. How does Cav-1 loss promote oxidative stress and mitochondrial dysfunction? Previous studies have found that Cav-1 loss has a close link with the increased NO synthase (NOS) activity and NO production (Figure 3). Although physiological NO plays significant role in maintaining cellular normal functions and modulating inflammatory response, excessive NO production is deleterious, as it inhibits mitochondrial OXPHOS chain and induces the overproduction of superoxide anion, hydrogen peroxide, and peroxynitrite, which exert potent cytotoxic and carcinogenic effects. For example, peroxynitrite could lead to mutation in the $p 53$ tumor suppressor gene and initiate oncogenic events [132]. NO is synthesized by at least four 


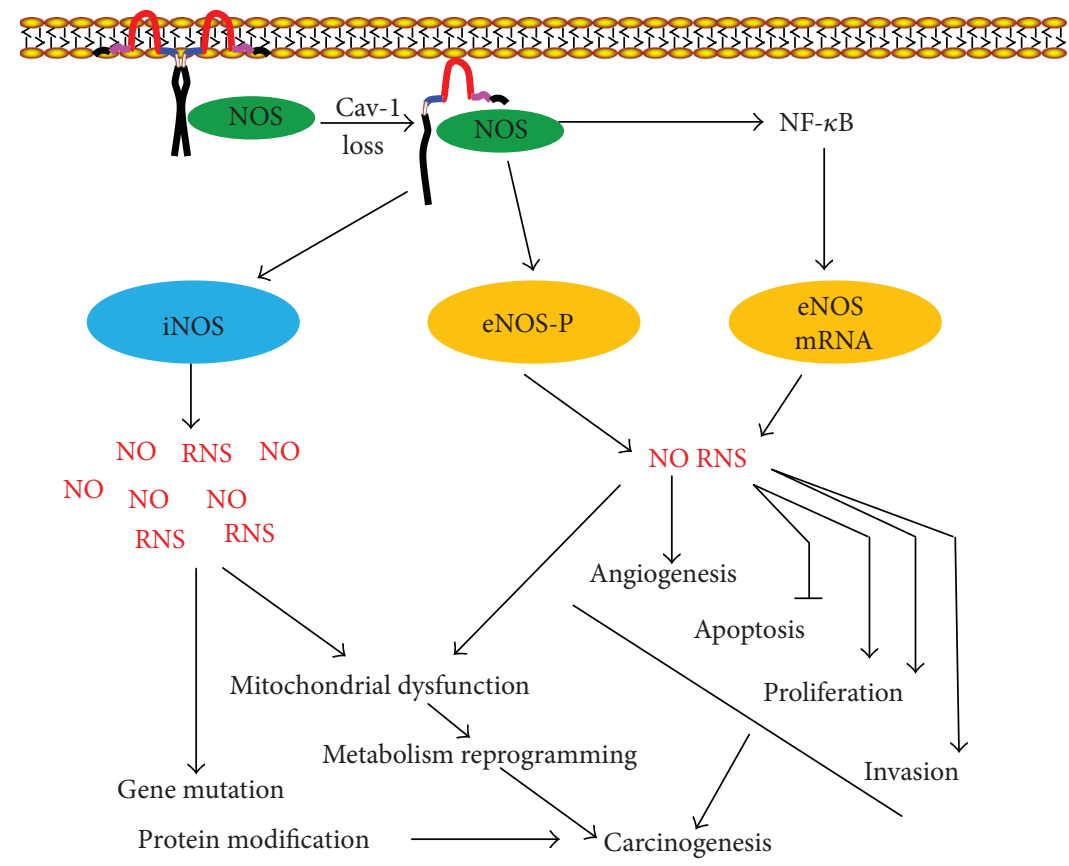

FIGURE 3: Cav-1 loss facilitates carcinogenesis through activating NOS activity. Cav-1 could directly interact with NOS enzymes through the scaffolding region. Following Cav-1 loss, eNOS will be released from the complex and activated through phosphorylation and mRNA overexpression, leading to the overproduction of NO and RNS. The high levels of NO and/or RNS would facilitate cell proliferation, apoptosis evasion, angiogenesis, and EMT process and finally induce carcinogenic transformation. Besides eNOS, iNOS will also be activated following Cav-1 loss. Since iNOS is capable of generating the micromolar level of NO, its activation will bring RNS burst and results in DNA damage and mitochondrial dysfunction, which finally promotes carcinogenesis.

isoforms of NOS enzyme: endothelial NOS (eNOS), inducible NOS (iNOS), neuronal NOS (nNOS), and, more recently, mitochondrial NOS. Mounting evidence has validated the increased NOS expression and/or activities, especially for the eNOS subtype, in human malignancies such as breast, central nervous system, and colon tumors [133]. eNOS was demonstrated to produce nanomolar level of $\mathrm{NO}$, and its activation was validated to promote cancer angiogenesis cascade, apoptosis evasion, and epithelialmensenchymal transition (EMT) process [134]. Interestingly, eNOS was demonstrated to directly interact with Cav-1 through its scaffolding domain [135]. Meanwhile, Cav-1 internalization has been shown to further regulate eNOS activation [136]. CAV1-deficient mice also displayed elevated eNOS expression and phosphorylation, and eNOS inhibitor could block Cav-1 loss-induced cancer growth, angiogenesis, and metastasis [137]. Since only iNOS produces micromolar NO concentrations, iNOS activation would bring much more influences on DNA damage and mitochondrial activity. Therefore, targeting iNOS for cancer prevention and treatment has also been extensively studied. iNOS expression was also revealed upregulated in multiple malignancies including colon, breast, prostate, bladder, and skin tumors [138]. Meanwhile, administration of iNOS selective inhibitor was also demonstrated to interrupt the development of AOM-induced aberrant crypt foci, accompanied by reduced expression of COX-2 and oxidative stress [139]. All these findings highlighted the significant role of NOS/ NO signaling in mediating Cav-1 anticarcinogenic activities.
Besides NOS/NO signaling, genetic profiling assay also showed that silencing CAV1 in stromal cells, 48 known ROS-related genes were upregulated, which are mainly associated with mitochondrial oxidative phosphorylation and peroxisome biogenesis. Furthermore, 45 HIF-target genes, 21 glycolytic enzymes, and $86 \mathrm{NF}-\kappa \mathrm{B}$-related genes were transcriptionally upregulated, implying that cellular metabolism was switched to glycolytic phenotype, which favors cancer initiation and growth [50]. ROS overproduction is closely associated with DNA damage response such as PARP. It was found that 5 PARP genes and 2 DNA-damage-induced transcripts Ddit3/Ddit4l were upregulated following CAV-1 knockdown [50]. Another genomic analysis also suggested that $C A V 1^{-l-}$ stromal cells showed the upregulation of 55 genes associated with oxidative stress, accompanied with overexpression of 129 genes correlated with DNA damage/ repair response [50]. All these results indicated that elevated ROS production following CAV1 silencing might facilitate cancer formation through activating DNA damage response. It is interesting to elucidate the precise interaction mode between Cav-1 and oxidative stress.

\section{Cav-1 and Oxidative Stress Regulation}

Increasing studies have proved that Cav-1 is an oxidative stress-related protein, as reactive species could affect the expression, degradation, posttranslational modifications, and membrane trafficking of Cav-1 (Figure 4). Meanwhile, modulating the Cav-1 pathway could significantly affect the 


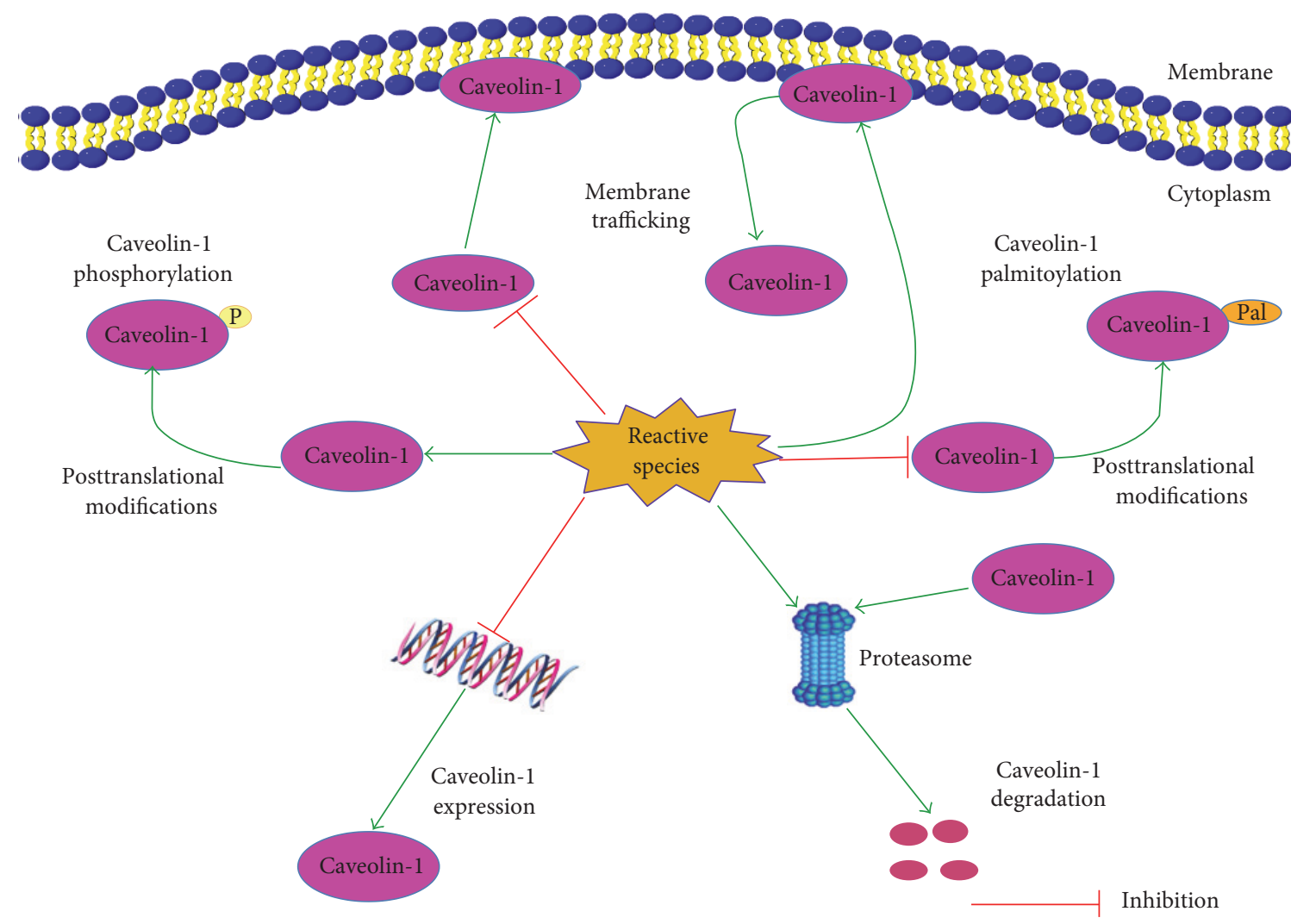

Figure 4: Cav-1 is an oxidative stress-related protein. On one hand, reactive species could decrease Cav-1 expression and induce its degradation through the proteasome pathway. On the other hand, reactive species could induce the phosphorylation but inhibit the palmitoylation of Cav-1. In addition, reactive species could induce Cav-1 internalization and inhibit the trafficking of newly synthesized Cav-1 to membrane raft domains.

oxidative stress status in normal and cancer cells. Therefore, Cav-1 may be a target of antioxidants in oxidative stress modulation and cancer antioxidant prevention. Herein, we summarize the available evidences about the implications of Cav-1 in the oxidative stress modulation effect of antioxidants and shed novel insights for Cav-1-targeted antioxidant therapy in cancer.

5.1. Cav-1 Is an Oxidative Stress-Related Protein. Intracellular reactive species are generated to serve as the second messengers, and some are linked to caveolae-signaling systems. Numerous cell surface receptors, which initiate a signal transduction cascade involving reactive species when activated by ligand binding, are recruited in caveolae [69]. These membrane microdomains, which play a pivotal role in signal transduction [140], therefore have been proposed to be a preferred site of reactive species generation [141].

5.1.1. Oxidative Stress Modulates the Expression and Degradation of Cav-1. Cav-1 reduction could significantly perturb the function of caveolae. In spite of Cav-1 reduction, caveolae are still able to be assembled at the plasma membrane, but their functions will be significantly impaired [142]. Oxidative stress could lead to the reduction of Cav-1 by modulating its expression and degradation (Figure 4).
For example, Cai et al. reported that inhibitors of ROS could increase the expression of Cav-1 in human brain tumor microvascular endothelial cells [143]. Zhang et al. reported that high concentrations of glucose could decrease Cav-1 expression in lens epithelial cells [144]. Furthermore, Mougeolle and colleagues reported that hydrogen peroxide at nontoxic concentrations could increase the concentrations of reactive species in myoblasts and decrease the expression level of Cav-1. However, this phenomenon was not observed in the presence of a proteasome inhibitor, suggesting that Cav-1 was rapidly degraded by the proteasome [142]. Moreover, Luanpitpong and colleagues have found that superoxide anion and hydrogen peroxide could attenuate the expression of Cav-1 in lung carcinoma H460 cells [145]. Further mechanism studies indicated that the downregulation effect of superoxide anion and hydrogen peroxide on Cav-1 is modulated through a protein degradation mechanism via the ubiquitin-proteasome pathway [145].

\subsubsection{Oxidative Stress and Posttranslational Modifications of} Cav-1. Cav-1 is mainly subject to two kinds of posttranslational modifications that regulate its activity, including phosphorylation and palmitoylation. Phosphorylation of Cav-1 is closely connected with cell apoptosis and cell attachment during oxidative stress. Increased expression of $\mathrm{p}-\mathrm{Cav}-1$ is 
antiapoptotic and may promote cell survival after oxidative stress [146, 147]. Cav-1 was first identified as a phosphoprotein in Rous sarcoma virus-transformed chicken embryo fibroblasts, which led to the hypothesis that Cav-1 may be a critical target during cellular transformation [148]. Since then, Cav-1 has been commonly identified as a phosphoprotein [56, 149-151]. Normally, Cav-1 is phosphorylated at a low $[149,150]$ or undetectable level $[56,151]$ in unstimulated cells. Reactive species generation could promote Cav-1 phosphorylation (Figure 4) [152]. For example, Volonte et al. suggested that multiple cellular stresses including high osmolarity, hydrogen peroxide, and UV light could induce the tyrosine 14-phosphorylation of Cav-1 [56]. Sun's group reported that ROS overproduction induced by high glucose-containing medium could time dependently increase Cav-1 phosphorylation in podocytes [153]. In addition, it was reported that an increased tyrosine-14 phosphorylation of Cav-1 was detected in human umbilical vein endothelial cells (HUVECs) after treatment with $100 \mu \mathrm{M}$ hydrogen peroxide for 30 minutes [154]. This increased tyrosine phosphorylation could be inhibited by tyrosine kinase inhibitors (herbimycin or genistein), which is consistent with the finding of Volonte [56]. What is more, Cav-1 could also be phosphorylated on serine-80 [155], and recent evidences suggested that serine phosphorylation of Cav-1 might be inversely correlated to tyrosine 14-phosphorylation [156].

Palmitoylation is the regulated, posttranslational modification of cysteine, serine, or threonine residues by the saturated fatty acid palmitate (e.g., palmitic acid), through a covalent thioester linkage [157]. Palmitoylation enhances the hydrophobicity of proteins and contributes to their membrane association. Palmitoylation typically occurs in membrane proteins and plays an important role in subcellular trafficking of proteins between membrane compartments, as well as in modulating protein-protein interactions [158]. Like many caveolae-targeted proteins, Cav-1 could also be acylated. Three cysteine residues near the C-terminus of Cav-1 (cysteines 133, 143, and 156) are susceptible to palmitoylation [159]. Cav-1 palmitoylation is required for cholesterol binding and transport to caveolae and for interaction with c-Src $[160,161]$. Mutation of the cysteine residues could impair Cav-1 interaction with other acylated proteins [161] and its binding and transport of cholesterol. It has been reported that reactive species, such as hydrogen peroxide, could markedly inhibit the palmitoylation of Cav-1 (Figure 4) [150]. Moreover, the effect of hydrogen peroxide on Cav-1 palmitoylation usually occurs at much lower concentrations than those required to affect Cav-1 phosphorylation, indicating that the effect on palmitoylation is not a direct consequence of the increased phosphorylation.

5.1.3. Oxidative Stress and the Membrane Trafficking of $C a v-1$. The function and activity of Cav-1 are tightly regulated by posttranscriptional modification as well as its subcellular localization. An early recognition that Cav-1 may be a sensitive target of oxidative stress modulation originated from the observation that oxidation of caveolar cholesterol by cholesterol oxidase could cause a reversible translocation of Cav-1 from the caveolae to the Golgi apparatus [162]. By applying immunofluorescence assay, Kang et al. have demonstrated that an exposure to high doses $(1 \mathrm{mM})$ of hydrogen peroxide could induce Cav-1 internalization in NIH3T3 fibroblasts [58]. Similarly, by carrying out biochemical experiments, Blair's group also found that treatment of endothelial cells with oxidized LDL, but not native LDL, could lead to the translocation of Cav-1 from plasma membrane caveolae to an intracellular membrane fraction [163]. In addition, metabolic labeling experiments also showed that hydrogen peroxide could inhibit the trafficking of newly synthesized Cav-1 to membrane raft domains in endothelial cells [150], and impairment of Cav-1 synthesis by hydrogen peroxide was not responsible for the diminished trafficking. Altogether, these results indicate that oxidative stress could affect the membrane trafficking of Cav-1 (Figure 4).

\subsection{The Regulation Effects of Cav-1 on Oxidative Stress. Cav-1} also has a feedback regulation effect on oxidative stress status in cells (Figure 5). Shiroto and colleagues found that CAV1 silencing in endothelial cells could increase the production of ROS in mitochondria and induce oxidative stress [164]. 2-deoxy-D-glucose, a glycolytic inhibitor, attenuated this increase, suggesting Cav-1 is in control of oxidative stress through glycolytic modulations [164]. Pavlides and colleagues have demonstrated that Cav-1 loss could induce oxidative stress, mimic hypoxia, and drive inflammation in the tumor microenvironment [50]. Martinez-Outschoorn et al. found that Cav-1 loss could lead to mitochondrial dysfunction, oxidative stress, and aerobic glycolysis in cancer-associated fibroblasts [60]. Wang et al. reported that Cav-1 knockdown resulted in eNOS redistribution to the perinuclear region and nearly doubled insulin-induced $\mathrm{NO}$ production in vascular endothelial cells [165]. Since NO is a competitive inhibitor of oxygen in the cytochrome oxidase present in mitochondrial complex IV, increased NO production could result in the inhibition of mitochondrion by attenuating the terminal phase of the mitochondrial electron transport chain complex, leading to electron leakage, superoxide formation, and mitochondrial dysfunction (Figure 5).

Cav-1 is highly expressed in endothelium. Therefore, the enriched Cav-1 in tumor vessels provides an interesting opportunity for Cav-1-targeted therapies. Gratton et al. demonstrated that a cell-permeable peptide derived from the CSD of Cav-1 could regulate microvascular permeability via inhibiting eNOS, and consequently markedly reduced tumor progression in mice [166]. Similarly, Suchaoin et al. reported that lung cancer $\mathrm{H} 460$ cells stably transfected with CAV1-overexpressing plasmids (H460/Cav-1) exhibited decreased ROS signal, while CAV1-specific shRNA-transfected (H460/shCav-1) cells showed enhanced ROS signal [52]. Interestingly, Pongjit et al. also established stable Cav-1-overexpressing (H460/Cav-1) cells and investigated the role of Cav-1 in modulating the oxidative stress induced by cisplatin. They found that overexpression of Cav-1 generated significantly higher superoxide anion level and could sensitize cisplatin-induced lung carcinoma cell apoptosis. Meanwhile, lung cancer H460 cells transfected with CAV1-specific shRNAs exhibited decreased 


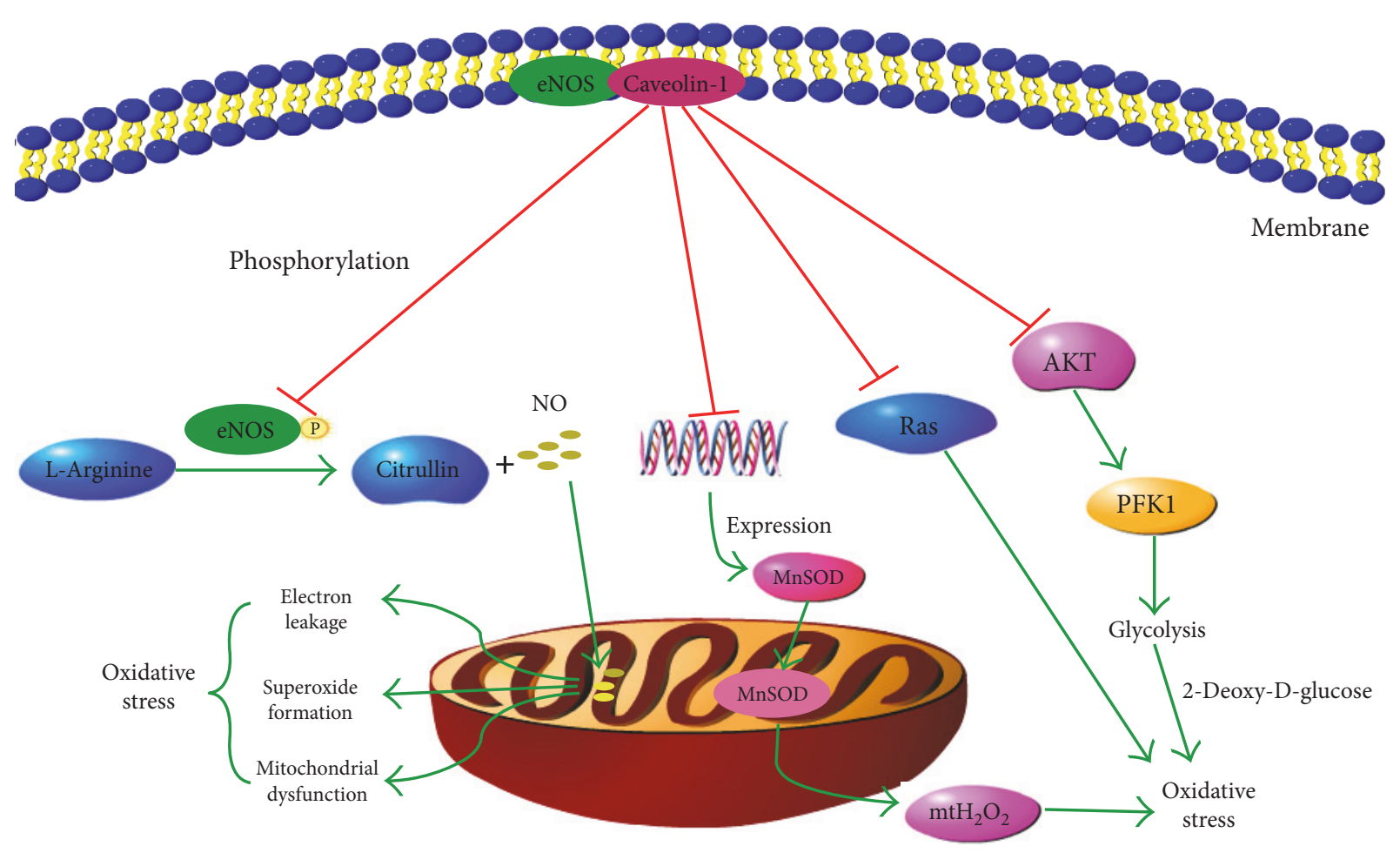

FIgURE 5: Regulation mechanisms of Cav-1 on oxidative stress. eNOS is maintained in an inactive state in caveolae by its interaction with Cav-1-scaffolding domain. Upon stimulation, eNOS is released and phosphorylated and could catalyze the NO synthesis reaction. NO overproduction could result in the inhibition of mitochondrion by attenuating the terminal phase of the electron transport chain complex, leading to electron leakage, superoxide formation, and mitochondrial dysfunction. Furthermore, Cav-1 could inhibit the transcription of $\mathrm{MnSOD}$ and thus reduce the release of excess mitochondria-derived $\mathrm{H}_{2} \mathrm{O}_{2}\left(\mathrm{mtH}_{2} \mathrm{O}_{2}\right)$. Moreover, glycolysis process could lead to the overproduction of reactive species, while 2-deoxy-D-glucose, a glycolysis inhibitor, could prevent reactive species burst. Phosphofructokinase 1 (PFK1) is the rate-limiting enzyme of the glycolysis process. Cav-1 could inactivate the PI3K/AKT/PFK1 pathway and therefore block the glycolysis process. Finally, Cav-1 could prevent the overproduction of reactive species by inactivating the Ras pathway.

superoxide generation and decreased cisplatin susceptibility [27]. Although these results revealed a heterogeneous outcome of CAV1 overexpression/silencing to oxidative stress modulation, they revealed the feedback regulation pattern between Cav-1 and oxidative stress.

5.3. Modulation Effects of Antioxidants on Cav-1. As described above, reactive species could modulate the expression, degradation, posttranslational modifications, and membrane trafficking of Cav-1, while Cav-1-targeted treatments could scavenge the reactive species. More importantly, emerging evidence has indicated that multiple antioxidants could exert antitumor activities in cancer cells as well as protective activities in normal cells by modulating the Cav-1 pathway (Figure 6).

In terms of cancer cells, Kitano et al. reported that vitamin $\mathrm{K} 3$ analogs could induce selective tumor cytotoxicity in neuroblastoma by inducing Cav-1 expression [59]. Yang et al. proved that resveratrol could induce cytotoxicity in a hepatocellular carcinoma model by increasing the expression of endogenous Cav-1 [167]. Salani et al. found that metformin exerted antiproliferative effect in NSCLC cells by inducing the expression of Cav-1 [61]. MartinezOutschoorn and colleagues reported that treatment with antioxidants (such as N-acetyl-cysteine, metformin, and quercetin) or NO inhibitors was sufficient to reverse many of the Cav-1 loss-induced phenotypes, such as mitochondrial dysfunction, oxidative stress, and aerobic glycolysis in cancer-associated fibroblasts [60].

In terms of normal cells, Zhan et al. reported that ginsenoside Rg1 exerted protective effect on bleomycin-induced pulmonary fibrosis in rats by increasing the expression of CAV1 mRNA and protein [168]. Nakaso et al. reported that knockdown of Cav-1 and/or Cav-2 prevented the protective effects of tocotrienol in a cellular Parkinson's disease model [169]. In addition, antioxidants such as lycopene [170] and quercetin [171] were also able to induce the expression of Cav-1. In particular, the effects of curcumin on Cav-1 pathway have been deeply reported. For example, Zeng et al. reported that curcumin exerted the antiproliferative effect in airway smooth muscle cells by upregulating the expression of Cav-1, while the disruption of caveolae using methyl- $\beta$ cyclodextrin attenuated the antiproliferative effects of curcumin [172]. Sun et al. reported that curcumin could prevent EMT of podocytes by suppressing the phosphorylation of Cav- 1 and increasing stabilization of Cav- 1 and $\beta$-catenin [173]. In addition, curcumin could inhibit renal inflammatory gene expression in vivo by reducing Cav-1 phosphorylation at Tyr (14) [174]. Yuan et al. investigated the mechanism of curcumin in increasing Cav-1 expression and found that 
<smiles>CC(C)=CCC[C@](C)(O[C@@H]1O[C@H](CO)[C@@H](O)[C@H](O)[C@H]1O)[C@H]1CC[C@]2(C)C1[C@@H](O)CC1[C@@]3(C)CC[C@H](O)C(C)(C)C3[C@@H](O[C@@H]3O[C@H](CO)[C@@H](O)[C@H](O)[C@H]3O)C[C@]12C</smiles>

Ginsenoside Rg1<smiles>CC1=CC(=O)c2ccccc2C1=O</smiles>

Vitamin K3<smiles>CN(C)C(=N)NC(=N)N</smiles>

Metformin<smiles>COc1cc(/C=C/C(=O)/C=C(O)/C=C/c2ccc(O)c(OC)c2)ccc1O</smiles>

Curcumin<smiles>CC(=O)N[C@@H](CS)C(=O)O</smiles>

N-Acetyl-cysteine<smiles>O=c1c(O)c(-c2ccc(O)c(O)c2)oc2cc(O)cc(O)c12</smiles>

Quercetin

Figure 6: Chemical structures of antioxidants influencing Cav-1 expression.

curcumin increased the expression of Cav-1 by inhibiting nuclear translocation of SREBP-1 [175]. In addition, curcumin could suppress ROS overproduction, which was believed to participate in blocking high-glucose-induced apoptosis of podocyte through regulating Cav-1 phosphorylation in both in vitro and in vivo experiments [153]. Altogether, the above evidences suggest that Cav-1 is a target of antioxidants in oxidative stress modulation, further proving that Cav-1 is an oxidative stress-related target for cancer antioxidant prevention.

Although antioxidant treatments have been explored for decades as attractive cancer prevention strategies, no antioxidant treatment regimen has reached the clinic at present. Notably, some preclinical and clinical trials even suggested that the cancer prevention potential of antioxidants was contrary to the expected outcome [176]. For example, Sayin and 
colleagues recently reported that supplementing the diet with antioxidants $\mathrm{N}$-acetylcysteine and vitamin E markedly increased tumor progression and reduced survival in mouse models of lung cancer [177]. The very large "Selenium and Vitamin E Cancer Prevention Trial" found no initial reduction in the risk of prostate cancer in healthy individuals taking either selenium or vitamin E supplements [178]. Indeed, long-term follow-up studies of these individuals suggested that vitamin E supplementation significantly increased the risk of prostate cancer among healthy men [179]. The disappointing results might be attributed to the multiple targets of antioxidants. Meanwhile, the heterogeneous in the dose, type, schedule, and action mechanisms of different antioxidant drugs might also have great influence on the endpoint. What is more, the poor solubility, stability, and limited bioavailability of some antioxidants also significantly impact on their therapeutic efficacy. Therefore, development of Cav-1-specific targeting antioxidant might be a novel strategy to improve the antioxidant prevention effects.

\section{Therapeutic Implication of Cav-1-Targeted Treatment for Cancer Antioxidant Prevention}

For many years, researchers have theorized that cancer cells depend on the activation of oncogenes or the inactivation of tumor suppressor genes for their survival. This theory is known as "oncogene addiction" [180]. However, recent studies have shed light on the vital mechanisms that ensure the survival of cancer cells, including the ability to escape from immune surveillance as well as the ability to undergo metabolic adaptations that provide cancer cells with a secure energy supply for their uncontrolled proliferation. Thus, targeting the "cart" (e.g., metabolism) rather than the "horse" (oncogenes and tumor suppressor genes) may be a more promising strategy for eliminating cancer cells while sparing normal cells [181]. Cancer cells undergo a metabolism switch, characterized by impaired mitochondrial oxidative phosphorylation [6] and increased aerobic glycolysis [7]. The metabolic reprogramming of malignant cells may be a "cart", and targeting the aberrant metabolism has been perceived as a more promising strategy for cancer prevention and treatment. As previously stated, aerobic glycolysis could lead to the overproduction of carcinogenic reactive species [7, 182], while Cav-1 could regulate cancer cell metabolism via suppressing MnSOD-driven glycolysis [183]. Meanwhile, Gene microarray analysis revealed that $C A V 1^{-1-}$ stromal cells showed the upregulation of 48 genes associated with ROS production, 45 genes regulated by HIF-1, and 21 genes involved in glycolysis pathway [184]. In addition, $C A V 1^{-1-}$ stromal cells exhibited defective mitochondrial functions due to the overexpression of NO [185]. Coculture of $C A \mathrm{V1}^{-/-}$stromal cells with cancer cells significantly promoted tumor growth and angiogenesis, while glycolysis inhibitor treatment greatly blocked the positive effects of $C A V 1^{-1-}$ stromal cells $[50,185]$. All these results indicated that Cav-1 may be a critical molecule in linking mitochondrial dysfunction, reactive species elevation, glycolysis enhancement, and finally carcinogenesis promotion. Therefore, therapeutic strategies restoring Cav-1 expression in both cancer cells and adjacent stromal cells should block cancer development via inhibiting glycolytic activity and reactive species overproduction.

On the other hand, compared with normal cells, cancer cells exhibit elevated levels of reactive species. Excessive reactive species not only induce oxidative damages and inactivation of tumor suppressor genes such as $p 53$ and PTEN but also activate the phosphorylation of Cav-1, which is reported to promote cancer cell growth and survival [186]. Tyrosine14 was documented to be the main phosphorylation site responsible for Cav-1 downstream signaling transduction. Therefore, it is feasible to apply tyrosine kinase inhibitors (TKIs) to interrupt Cav-1 phosphorylation and finally inhibit ROS-induced carcinogenesis. Gefitinib, one kind of TKIs for advanced non-small-cell lung cancer therapy, was reported to inhibit EGF-induced stimulation of both EGFR and downstream Akt and MAPK more efficiently in MCF-7 cells overexpressing Cav-1 than in parental cells [187]. Similarly, the level of phosphorylated Cav-1 was also upregulated in drug-resistant BT474 cells but was blocked by lapatinib [188]. Meanwhile, ovarian cancer cells with high expression of Cav-1 were particularly sensitive to dasatinib [189]. Intriguingly, several TKIs were demonstrated to be effective in preventing carcinogenesis. For example, gefitinib was validated to reduce mammary tumor incidence by $50 \%$ [190]. Lapatinib and pazopanib were also demonstrated effective in preventing breast cancer metastasis [191, 192]. What is more is that sorafenib was suggested to be applied for preventing hepatocellular carcinoma recurrence after liver transplantation [193]. All these findings implied that Cav-1 phosphorylation inhibition might be a promising strategy for cancer prevention, and it is of great value to develop Cav-1-targeted TKI for cancer prevention.

The carcinogenic properties of excessive reactive species have prompted the evaluation of dietary antioxidant supplementations as potential cancer preventive agents. However, the preventive efficacies of dietary antioxidant supplementations are disappointing in the current clinical trials. Based on the understanding that mitochondrion is the main source of reactive species in cancer cells, therapies that directly inhibit the production of mitochondrial-derived reactive species, or that scavenge reactive species in mitochondrion, will be more effective than dietary antioxidants, because the latter poorly access the mitochondrial-localized pools of reactive species. Therefore, development of mitochondrion-targeting antioxidants delivery system is a better therapeutic approach for cancer antioxidant prevention. To date, a variety of mitochondrion-targeting antioxidants are being developed, and the best characterized one is $10-\left(6^{\prime}\right.$-ubiquinonyl) decyltriphenylphosphonium, which could specifically accumulate in the mitochondrial matrix by several hundredfold because of the large mitochondrial transmembrane potential [194]. Caveolae are not the preferred location for Cav-1 in all cell types; instead, Cav-1 can be targeted to a variety of intracellular destinations, particularly in the mitochondrion [21, 22]. CAV1-null MEFs (mouse embryonic fibroblasts) had a higher mitochondrial membrane potential and a 
preference for glycolysis. Meanwhile, the circulating $\mathrm{H}_{2} \mathrm{O}_{2}$ and pyruvate levels in $\mathrm{CAVI}^{-1-}$ mice were significantly elevated, indicating the altered mitochondrial function and metabolic inflexibility associated with the loss of Cav-1 [195]. Another study also demonstrated that Cav-1 deficiency could increase the mitochondrial membrane condensation, accompanied with dysfunction of respiratory chain efficiency and intrinsic antioxidant defense [196]. Mechanism study further validated that Cav-1 loss resulted in the cytoplasmic and proteasome-dependent degradation of complexes I, III, IV, and V but had no effects on either mitochondrial number or morphology [197]. All these findings suggested that strategies targeting Cav-1 translocation to mitochondrion might be a novel approach for cancer prevention. It is worth to study the detailed molecular mechanisms underlying the targeted delivery of Cav-1 to mitochondrion, and it is anticipated to see enriched mitochondrial Cav-1 expression would decrease the incidence of malignant transformation via inhibiting reactive species overproduction.

\section{Conclusions and Perspectives}

As stated above, both oxidative stress and Cav-1 are closely connected with the tumorigenesis and progression of cancer. Compared with normal cells, cancer cells usually demonstrate aberrations in oxidative metabolism and signaling pathways, characterized by increased levels of reactive species. Reactive species overproduction could induce tumorigenesis and progression possibly by modulating the expression, degradation, posttranslational modifications (including tyrosine phosphorylation and palmitoylation), and subcellular localization of Cav-1. Meanwhile, Cav-1 mainly acts as a tumor suppressor during cancer initiation and development and also has a feedback regulation effect on oxidative stress. More importantly, emerging evidences have indicated that multiple antioxidants could exert antitumor activities in cancer cells as well as protective activities in normal cells by modulating the Cav-1 pathway. Altogether, these observations suggest that Cav-1 may be a potential oxidative stress-related protein for cancer antioxidant prevention. However, it should be noted that existing studies concerning the interaction between Cav-1 and reactive species were mainly applied in normal endothelial or epithelial cells, but less in cancer cells. Therefore, more research focusing on cancer samples are urgent for a better understanding the role of Cav-1 in oxidative stress-induced cancer initiation and progression, as well as its potential as an oxidative stressrelated protein for cancer antioxidant prevention. What is more, development of Cav-1-targeting strategies and antioxidant drugs might shed novel light on cancer prevention and antioxidant research.

In summary, this review reveals the roles of Cav-1 and oxidative stress in tumorigenesis and progression as well as their interaction, proposing Cav- 1 as a promising candidate target for cancer antioxidant prevention, possibly providing novel insights for Cav-1-targeted strategies for cancer prevention and treatment.

\section{Conflicts of Interest}

The authors declare no conflicts of interest.

\section{Acknowledgments}

This work was supported by the National Natural Science Foundation of China (81402173, 81573651), Pearl River S\&T Nova Program of Guangzhou (201506010098), Combined Scientific Project Funded by Guangdong Provincial Science and Technology Agency and Guangdong Provincial Academy of Traditional Chinese Medicine (2014A020221047), Guangdong High-Level University Construction Project (A1-AFD018161Z1510), Guangdong High-Level Personnel of Special Support Program (A1-3002-16-111-003), International Postdoctoral Exchange Fellowship Program and China Postdoctoral Science Foundation (2016M592585), and GZUCM Science Fund for Creative Research Groups (2016KYTD10) and GZUCM Torch Program (XH20140108).

\section{References}

[1] R. K. Gupta, A. K. Patel, N. Shah et al., "Oxidative stress and antioxidants in disease and cancer: a review," Asian Pacific Journal of Cancer Prevention : APJCP, vol. 15, no. 11, pp. 4405-4409, 2014.

[2] J. E. Klaunig, L. M. Kamendulis, and B. A. Hocevar, "Oxidative stress and oxidative damage in carcinogenesis," Toxicologic Pathology, vol. 38, no. 1, pp. 96-109, 2010.

[3] T. B. Kryston, A. B. Georgiev, P. Pissis, and A. G. Georgakilas, "Role of oxidative stress and DNA damage in human carcinogenesis," Mutation Research, vol. 711, no. 1-2, pp. 193-201, 2011.

[4] J. E. Klaunig, Z. Wang, X. Pu, and S. Zhou, "Oxidative stress and oxidative damage in chemical carcinogenesis," Toxicology and Applied Pharmacology, vol. 254, no. 2, pp. 86-99, 2011.

[5] T. Finkel, "Signal transduction by reactive oxygen species," The Journal of Cell Biology, vol. 194, no. 1, pp. 7-15, 2011.

[6] Y. Yang, S. Karakhanova, W. Hartwig et al., "Mitochondria and mitochondrial ROS in cancer: novel targets for anticancer therapy," Journal of Cellular Physiology, vol. 231, no. 12, pp. 2570-2581, 2016.

[7] R. A. Cairns, I. S. Harris, and T. W. Mak, "Regulation of cancer cell metabolism," Nature Reviews. Cancer, vol. 11, no. 2, pp. 85-95, 2011.

[8] M. Sundaresan, Z. X. Yu, V. J. Ferrans et al., "Regulation of reactive-oxygen-species generation in fibroblasts by Rac1," The Biochemical Journal, vol. 318, no. Part 2, pp. 379-382, 1996.

[9] V. Nogueira, Y. Park, C. C. Chen et al., "Akt determines replicative senescence and oxidative or oncogenic premature senescence and sensitizes cells to oxidative apoptosis," Cancer Cell, vol. 14, no. 6, pp. 458-470, 2008.

[10] F. Hecht, C. F. Pessoa, L. B. Gentile, D. Rosenthal, D. P. Carvalho, and R. S. Fortunato, "The role of oxidative stress on breast cancer development and therapy," Tumour Biology, vol. 37, no. 4, pp. 4281-4291, 2016.

[11] P. A. Thompson, E. L. Ashbeck, D. J. Roe et al., "Selenium supplementation for prevention of colorectal adenomas and 
risk of associated type 2 diabetes," Journal of the National Cancer Institute, vol. 108, no. 12, 2016.

[12] K. N. Prasad, "Simultaneous activation of Nrf2 and elevation of dietary and endogenous antioxidant chemicals for cancer prevention in humans," Journal of the American College of Nutrition, vol. 35, no. 2, pp. 175-184, 2016.

[13] K. C. Rocha, M. L. Vieira, R. L. Beltrame et al., "Impact of selenium supplementation in neutropenia and immunoglobulin production in childhood cancer patients," Journal of Medicinal Food, vol. 19, no. 6, pp. 560-568, 2016.

[14] N. S. Chandel and D. A. Tuveson, "The promise and perils of antioxidants for cancer patients," The New England Journal of Medicine, vol. 371, no. 2, pp. 177-178, 2014.

[15] J. X. Chen, G. Li, H. Wang et al., "Dietary tocopherols inhibit PhIP-induced prostate carcinogenesis in CYP1A-humanized mice," Cancer Letters, vol. 371, no. 1, pp. 71-78, 2016.

[16] C. Gorrini, I. S. Harris, and T. W. Mak, "Modulation of oxidative stress as an anticancer strategy," Nature Reviews. Drug Discovery, vol. 12, no. 12, pp. 931-947, 2013.

[17] H. Greenlee, D. L. Hershman, and J. S. Jacobson, "Use of antioxidant supplements during breast cancer treatment: a comprehensive review," Breast Cancer Research and Treatment, vol. 115, no. 3, pp. 437-452, 2009.

[18] P. J. Smith and K. J. Steadman, “Antioxidant supplementation and cancer patients receiving curative-intent chemotherapy," The Medical Journal of Australia, vol. 204, no. 5, pp. 185-185e1, 2016.

[19] M. K. Chung, D. H. Kim, Y. C. Ahn, J. Y. Choi, E. H. Kim, and Y. I. Son, "Randomized trial of vitamin C/E complex for prevention of radiation-induced xerostomia in patients with head and neck cancer," Otolaryngology Head and Neck Surgery, vol. 155, no. 3, pp. 423-430, 2016.

[20] J. P. Cheng and B. J. Nichols, "Caveolae: one function or many?” Trends in Cell Biology, vol. 26, no. 3, pp. 177-189, 2016.

[21] M. Sargiacomo, P. E. Scherer, Z. Tang et al., "Oligomeric structure of caveolin: implications for caveolae membrane organization," Proceedings of the National Academy of Sciences of the United States of America, vol. 92, no. 20, pp. 9407-9411, 1995.

[22] H. N. Fridolfsson, D. M. Roth, P. A. Insel, and H. H. Patel, "Regulation of intracellular signaling and function by caveolin," FASEB Journal, vol. 28, no. 9, pp. 3823-3831, 2014.

[23] M. Salatino, W. Beguelin, M. G. Peters et al., "Progestininduced caveolin-1 expression mediates breast cancer cell proliferation," Oncogene, vol. 25, no. 59, pp. 7723-7739, 2006.

[24] C. Du, L. Chen, H. Zhang et al., "Caveolin-1 limits the contribution of BKCa channel to MCF-7 breast cancer cell proliferation and invasion," International Journal of Molecular Sciences, vol. 15, no. 11, pp. 20706-20722, 2014.

[25] Y. Tang, X. Zeng, F. He, Y. Liao, N. Qian, and M. Toi, "Caveolin-1 is related to invasion, survival, and poor prognosis in hepatocellular cancer," Medical Oncology, vol. 29, no. 2, pp. 977-984, 2012.

[26] T. C. Thompson, T. L. Timme, L. Li, and A. Goltsov, "Caveolin-1, a metastasis-related gene that promotes cell survival in prostate cancer," Apoptosis, vol. 4, no. 4, pp. 233-237, 1999.

[27] K. Pongit and P. Chanvorachote, "Caveolin-1 sensitizes cisplatin-induced lung cancer cell apoptosis via superoxide anion-dependent mechanism," Molecular and Cellular Biochemistry, vol. 358, no. 1-2, pp. 365-373, 2011.
[28] L. Xu, X. Qu, H. Li et al., "Src/caveolin-1-regulated EGFR activation antagonizes TRAIL-induced apoptosis in gastric cancer cells," Oncology Reports, vol. 32, no. 1, pp. 318324, 2014

[29] R. Wang, Z. Li, H. Guo et al., "Caveolin 1 knockdown inhibits the proliferation, migration and invasion of human breast cancer BT474 cells," Molecular Medicine Reports, vol. 9, no. 5, pp. 1723-1728, 2014.

[30] L. Miao, X. Xiong, Y. Lin et al., "miR-203 inhibits tumor cell migration and invasion via caveolin-1 in pancreatic cancer cells," Oncology Letters, vol. 7, no. 3, pp. 658-662, 2014.

[31] M. Joglekar, W. O. Elbazanti, M. D. Weitzman, H. L. Lehman, and K. L. van Golen, "Caveolin-1 mediates inflammatory breast cancer cell invasion via the Akt1 pathway and RhoC GTPase," Journal of Cellular Biochemistry, vol. 116, no. 6, pp. 923-933, 2015.

[32] H. Yang, L. Guan, S. Li et al., "Mechanosensitive caveolin-1 activation-induced PI3K/Akt/mTOR signaling pathway promotes breast cancer motility, invadopodia formation and metastasis in vivo," Oncotarget, vol. 7, no. 13, pp. 16227-16247, 2016.

[33] L. Nimri, H. Barak, L. Graeve, and B. Schwartz, "Restoration of caveolin-1 expression suppresses growth, membrane-type4 metalloproteinase expression and metastasis-associated activities in colon cancer cells," Molecular Carcinogenesis, vol. 52, no. 11, pp. 859-870, 2013.

[34] Y. Shi, S. H. Tan, S. Ng et al., "Critical role of CAV1/caveolin1 in cell stress responses in human breast cancer cells via modulation of lysosomal function and autophagy," Autophagy, vol. 11, no. 5, pp. 769-784, 2015.

[35] R. Wang, W. He, Z. Li, W. Chang, Y. Xin, and T. Huang, "Caveolin-1 functions as a key regulator of 17beta-estradiolmediated autophagy and apoptosis in BT474 breast cancer cells," International Journal of Molecular Medicine, vol. 34, no. 3, pp. 822-827, 2014.

[36] Z.Xie, X.Zeng, T. Waldman, and R. I. Glazer, “Transformation of mammary epithelial cells by 3-phosphoinositide-dependent protein kinase-1 activates beta-catenin and c-Myc, and down-regulates caveolin-1," Cancer Research, vol. 63, no. 17 , pp. 5370-5375, 2003.

[37] P. Chunhacha and P. Chanvorachote, "Roles of caveolin-1 on anoikis resistance in non small cell lung cancer," International Journal of Physiology Pathophysiology and Pharmacology, vol. 4, no. 3, pp. 149-155, 2012.

[38] H. Halim, S. Luanpitpong, and P. Chanvorachote, "Acquisition of anoikis resistance up-regulates caveolin-1 expression in human non-small cell lung cancer cells," Anticancer Research, vol. 32, no. 5, pp. 1649-1658, 2012.

[39] W. Zou, X. Ma, W. Hua, B. Chen, and G. Cai, "Caveolin-1 mediates chemoresistance in cisplatin-resistant ovarian cancer cells by targeting apoptosis through the Notch-1/Akt/ NF-kappaB pathway," Oncology Reports, vol. 34, no. 6, pp. 3256-3263, 2015.

[40] S. C. Sekhar, T. Kasai, A. Satoh et al., "Identification of caveolin-1 as a potential causative factor in the generation of trastuzumab resistance in breast cancer cells," Journal of Cancer, vol. 4, no. 5, pp. 391-401, 2013.

[41] K. N. Wu, M. Queenan, J. R. Brody et al., "Loss of stromal caveolin-1 expression in malignant melanoma metastases predicts poor survival," Cell Cycle, vol. 10, no. 24, pp. 4250-4255, 2011. 
[42] A. K. Witkiewicz, A. Dasgupta, S. Sammons et al., "Loss of stromal caveolin-1 expression predicts poor clinical outcome in triple negative and basal-like breast cancers," Cancer Biology \& Therapy, vol. 10, no. 2, pp. 135-143, 2010.

[43] A. K. Witkiewicz, A. Dasgupta, F. Sotgia et al., "An absence of stromal caveolin-1 expression predicts early tumor recurrence and poor clinical outcome in human breast cancers," The American Journal of Pathology, vol. 174, no. 6, pp. 2023-2034, 2009.

[44] Z. Zhao, F. H. Han, S. B. Yang, L. X. Hua, J. H. Wu, and W. H. Zhan, "Loss of stromal caveolin-1 expression in colorectal cancer predicts poor survival," World Journal of Gastroenterology, vol. 21, no. 4, pp. 1140-1147, 2015.

[45] W. Shan-Wei, X. Kan-Lun, R. Shu-Qin, Z. Li-Li, and C. Li-Rong, "Overexpression of caveolin-1 in cancer-associated fibroblasts predicts good outcome in breast cancer," Breast Care, vol. 7, no. 6, pp. 477-483, 2012.

[46] K. C. Moon, G. K. Lee, S. H. Yoo et al., "Expression of caveolin-1 in pleomorphic carcinoma of the lung is correlated with a poor prognosis," Anticancer Research, vol. 25, no. 6C, pp. 4631-4637, 2005.

[47] J. A. Karam, Y. Lotan, C. G. Roehrborn, R. Ashfaq, P. I. Karakiewicz, and S. F. Shariat, "Caveolin-1 overexpression is associated with aggressive prostate cancer recurrence," The Prostate, vol. 67, no. 6, pp. 614-622, 2007.

[48] S. A. Tahir, G. Yang, S. Ebara et al., "Secreted caveolin-1 stimulates cell survival/clonal growth and contributes to metastasis in androgen-insensitive prostate cancer," Cancer Research, vol. 61, no. 10, pp. 3882-3885, 2001.

[49] L. Liu, H. X. Xu, W. Q. Wang et al., "Cavin-1 is essential for the tumor-promoting effect of caveolin-1 and enhances its prognostic potency in pancreatic cancer," Oncogene, vol. 33, no. 21, pp. 2728-2736, 2014.

[50] S. Pavlides, A. Tsirigos, I. Vera et al., "Loss of stromal caveolin-1 leads to oxidative stress, mimics hypoxia and drives inflammation in the tumor microenvironment, conferring the "reverse Warburg effect": a transcriptional informatics analysis with validation," Cell Cycle, vol. 9, no. 11, pp. 2201-2219, 2010.

[51] T. Songserm, V. Pongrakhananon, and P. Chanvorachote, "Sub-toxic cisplatin mediates anoikis resistance through hydrogen peroxide-induced caveolin-1 up-regulation in non-small cell lung cancer cells," Anticancer Research, vol. 32, no. 5, pp. 1659-1669, 2012.

[52] W. Suchaoin and P. Chanvorachote, "Caveolin-1 attenuates hydrogen peroxide-induced oxidative damage to lung carcinoma cells," Anticancer Research, vol. 32, no. 2, pp. 483-490, 2012.

[53] D. Volonte, K. Zhang, M. P. Lisanti, and F. Galbiati, "Expression of caveolin-1 induces premature cellular senescence in primary cultures of murine fibroblasts," Molecular Biology of the Cell, vol. 13, no. 7, pp. 2502-2517, 2002.

[54] P. Rungtabnapa, U. Nimmannit, H. Halim, Y. Rojanasakul, and P. Chanvorachote, "Hydrogen peroxide inhibits nonsmall cell lung cancer cell anoikis through the inhibition of caveolin-1 degradation," American Journal of Physiology. Cell Physiology, vol. 300, no. 2, pp. C235-C245, 2011.

[55] Y. N. Kim, G. J. Wiepz, A. G. Guadarrama, and P. J. Bertics, "Epidermal growth factor-stimulated tyrosine phosphorylation of caveolin-1. Enhanced caveolin-1 tyrosine phosphorylation following aberrant epidermal growth factor receptor status," The Journal of Biological Chemistry, vol. 275, no. 11, pp. 7481-7491, 2000.

[56] D. Volonte, F. Galbiati, R. G. Pestell, and M. P. Lisanti, "Cellular stress induces the tyrosine phosphorylation of caveolin-1 (Tyr(14)) via activation of p38 mitogen-activated protein kinase and c-Src kinase. Evidence for caveolae, the actin cytoskeleton, and focal adhesions as mechanical sensors of osmotic stress," The Journal of Biological Chemistry, vol. 276, no. 11, pp. 8094-8103, 2001.

[57] A. R. Sanguinetti and C. C. Mastick, "c-Abl is required for oxidative stress-induced phosphorylation of caveolin-1 on tyrosine 14," Cellular Signalling, vol. 15, no. 3, pp. 289-298, 2003.

[58] Y. S. Kang, Y. G. Ko, and J. S. Seo, "Caveolin internalization by heat shock or hyperosmotic shock," Experimental Cell Research, vol. 255, no. 2, pp. 221-228, 2000.

[59] T. Kitano, H. Yoda, K. Tabata et al., "Vitamin K3 analogs induce selective tumor cytotoxicity in neuroblastoma," Biological \& Pharmaceutical Bulletin, vol. 35, no. 4, pp. 617623, 2012.

[60] U. E. Martinez-Outschoorn, R. M. Balliet, D. B. Rivadeneira et al., "Oxidative stress in cancer associated fibroblasts drives tumor-stroma co-evolution: a new paradigm for understanding tumor metabolism, the field effect and genomic instability in cancer cells," Cell Cycle, vol. 9, no. 16, pp. 3256-3276, 2010.

[61] B. Salani, S. Maffioli, M. Hamoudane et al., "Caveolin-1 is essential for metformin inhibitory effect on IGF1 action in non-small-cell lung cancer cells," FASEB Journal, vol. 26, no. 2, pp. 788-798, 2012.

[62] U. E. Martinez-Outschoorn, F. Sotgia, and M. P. Lisanti, "Caveolae and signalling in cancer," Nature Reviews. Cancer, vol. 15, no. 4, pp. 225-237, 2015.

[63] J. R. Glenney Jr. and D. Soppet, "Sequence and expression of caveolin, a protein component of caveolae plasma membrane domains phosphorylated on tyrosine in Rous sarcoma virustransformed fibroblasts," Proceedings of the National Academy of Sciences of the United States of America, vol. 89, no. 21, pp. 10517-10521, 1992.

[64] A. Schlegel and M. P. Lisanti, "A molecular dissection of caveolin-1 membrane attachment and oligomerization. Two separate regions of the caveolin-1 C-terminal domain mediate membrane binding and oligomer/oligomer interactions in vivo," The Journal of Biological Chemistry, vol. 275, no. 28, pp. 21605-21617, 2000.

[65] P. Dupree, R. G. Parton, G. Raposo, T. V. Kurzchalia, and K. Simons, "Caveolae and sorting in the trans-Golgi network of epithelial cells," The EMBO Journal, vol. 12, no. 4, pp. 1597-1605, 1993.

[66] J. Lee and K. J. Glover, "The transmembrane domain of caveolin-1 exhibits a helix-break-helix structure," Biochimica et Biophysica Acta, vol. 1818, no. 5, pp. 1158-1164, 2012.

[67] P. E. Scherer, Z. Tang, M. Chun, M. Sargiacomo, H. F. Lodish, and M. P. Lisanti, "Caveolin isoforms differ in their Nterminal protein sequence and subcellular distribution. Identification and epitope mapping of an isoform-specific monoclonal antibody probe," The Journal of Biological Chemistry, vol. 270, no. 27, pp. 16395-16401, 1995.

[68] M. Simionescu, N. Simionescu, and G. E. Palade, "Biochemically differentiated microdomains of the cell surface of capillary endothelium," Annals of the New York Academy of Sciences, vol. 401, no. 1, pp. 9-24, 1982. 
[69] M. P. Lisanti, P. E. Scherer, J. Vidugiriene et al., "Characterization of caveolin-rich membrane domains isolated from an endothelial-rich source: implications for human disease," The Journal of Cell Biology, vol. 126, no. 1, pp. 111-126, 1994.

[70] H. H. Patel, F. Murray, and P. A. Insel, "Caveolae as organizers of pharmacologically relevant signal transduction molecules," Annual Review of Pharmacology and Toxicology, vol. 48, pp. 359-391, 2008.

[71] C. Mineo, G. L. James, E. J. Smart, and R. G. Anderson, "Localization of epidermal growth factor-stimulated Ras/ Raf-1 interaction to caveolae membrane," The Journal of Biological Chemistry, vol. 271, no. 20, pp. 11930-11935, 1996.

[72] J. Couet, S. Li, T. Okamoto, T. Ikezu, and M. P. Lisanti, "Identification of peptide and protein ligands for the caveolinscaffolding domain. Implications for the interaction of caveolin with caveolae-associated proteins," The Journal of Biological Chemistry, vol. 272, no. 10, pp. 6525-6533, 1997.

[73] M. Yamamoto, Y. Toya, C. Schwencke, M. P. Lisanti, M. G. Myers Jr., and Y. Ishikawa, "Caveolin is an activator of insulin receptor signaling," The Journal of Biological Chemistry, vol. 273, no. 41, pp. 26962-26968, 1998.

[74] V. J. Venema, R. Zou, H. Ju, M. B. Marrero, and R. C. Venema, "Caveolin-1 detergent solubility and association with endothelial nitric oxide synthase is modulated by tyrosine phosphorylation," Biochemical and Biophysical Research Communications, vol. 236, no. 1, pp. 155-161, 1997.

[75] M. Murata, J. Peranen, R. Schreiner, F. Wieland, T. V. Kurzchalia, and K. Simons, "VIP21/caveolin is a cholesterol-binding protein," Proceedings of the National Academy of Sciences of the United States of America, vol. 92, no. 22, pp. 10339-10343, 1995.

[76] A. Pol, R. Luetterforst, M. Lindsay, S. Heino, E. Ikonen, and R. G. Parton, "A caveolin dominant negative mutant associates with lipid bodies and induces intracellular cholesterol imbalance," The Journal of Cell Biology, vol. 152, no. 5, pp. 1057-1070, 2001.

[77] A. Tagawa, A. Mezzacasa, A. Hayer, A. Longatti, L. Pelkmans, and A. Helenius, "Assembly and trafficking of caveolar domains in the cell: caveolae as stable, cargo-triggered, vesicular transporters," The Journal of Cell Biology, vol. 170, no. 5, pp. 769-779, 2005.

[78] C. Boscher and I. R. Nabi, "Caveolin-1: role in cell signaling," Advances in Experimental Medicine and Biology, vol. 729, pp. 29-50, 2012.

[79] N. Patani, L. A. Martin, J. S. Reis-Filho, and M. Dowsett, “The role of caveolin-1 in human breast cancer," Breast Cancer Research and Treatment, vol. 131, no. 1, pp. 1-15, 2012.

[80] J. C. Zenklusen, J. N. Weitzel, H. G. Ball, and C. J. Conti, "Allelic loss at 7q31.1 in human primary ovarian carcinomas suggests the existence of a tumor suppressor gene," Oncogene, vol. 11, no. 2, pp. 359-363, 1995.

[81] J. A. Engelman, X. L. Zhang, and M. P. Lisanti, “Genes encoding human caveolin-1 and -2 are co-localized to the D7S522 locus (7q31.1), a known fragile site (FRA7G) that is frequently deleted in human cancers," FEBS Letters, vol. 436, no. 3, pp. 403-410, 1998.

[82] W. Ma, D. D. Wang, L. Li et al., "Caveolin-1 plays a key role in the oleanolic acid-induced apoptosis of HL-60 cells," Oncology Reports, vol. 32, no. 1, pp. 293-301, 2014.

[83] T. M. Williams and M. P. Lisanti, "Caveolin-1 in oncogenic transformation, cancer, and metastasis," American
Journal of Physiology. Cell Physiology, vol. 288, no. 3, pp. C494-C506, 2005.

[84] C. Caliceti, L. Zambonin, B. Rizzo et al., "Role of plasma membrane caveolae/lipid rafts in VEGF-induced redox signaling in human leukemia cells," BioMed Research International, vol. 2014, Article ID 857504, p. 13, 2014.

[85] K. Zhang, G. Yang, W. Wu et al., "Decreased expression of caveolin-1 and E-cadherin correlates with the clinicopathologic features of gastric cancer and the EMT process," Recent Patents on Anti-Cancer Drug Discovery, vol. 11, no. 2, pp. 236-244, 2016.

[86] X. J. Shen, H. Zhang, G. S. Tang et al., "Caveolin-1 is a modulator of fibroblast activation and a potential biomarker for gastric cancer," International Journal of Biological Sciences, vol. 11, no. 4, pp. 370-379, 2015.

[87] E. Burgermeister, T. Friedrich, I. Hitkova et al., "The Ras inhibitors caveolin-1 and docking protein 1 activate peroxisome proliferator-activated receptor gamma through spatial relocalization at helix 7 of its ligand-binding domain," Molecular and Cellular Biology, vol. 31, no. 16, pp. 3497-3510, 2011.

[88] P. Hammarsten, T. Dahl Scherdin, C. Hagglof et al., "High caveolin-1 expression in tumor stroma is associated with a favourable outcome in prostate cancer patients managed by watchful waiting," PloS One, vol. 11, no. 10, article e0164016, 2016.

[89] J. Huertas-Martinez, S. Rello-Varona, D. Herrero-Martin et al., "Caveolin-1 is down-regulated in alveolar rhabdomyosarcomas and negatively regulates tumor growth," Oncotarget, vol. 5, no. 20, pp. 9744-9755, 2014.

[90] F. Han and H. G. Zhu, "Caveolin-1 regulating the invasion and expression of matrix metalloproteinase (MMPs) in pancreatic carcinoma cells," The Journal of Surgical Research, vol. 159, no. 1, pp. 443-450, 2010.

[91] F. Han and H. G. Zhu, "Over-expression of caveolin-1 inhibits proliferation and invasion of pancreatic carcinoma cells in vitro," Zhonghua Zhong Liu Za Zhi, vol. 31, no. 10, pp. 732-737, 2009.

[92] S. F. Chen, J. Y. Liou, T. Y. Huang et al., "Caveolin-1 facilitates cyclooxygenase-2 protein degradation," Journal of Cellular Biochemistry, vol. 109, no. 2, pp. 356-362, 2010.

[93] R. Feldman and J. D. Martinez, "Growth suppression by ursodeoxycholic acid involves caveolin-1 enhanced degradation of EGFR," Biochimica et Biophysica Acta, vol. 1793, no. 8, pp. 1387-1394, 2009.

[94] C. Trimmer, D. Whitaker-Menezes, G. Bonuccelli et al., "CAV1 inhibits metastatic potential in melanomas through suppression of the integrin/Src/FAK signaling pathway," Cancer Research, vol. 70, no. 19, pp. 74897499, 2010.

[95] J. Kang, J. H. Park, H. J. Lee et al., "Caveolin-1 modulates docetaxel-induced cell death in breast cancer cell subtypes through different mechanisms," Cancer Research and Treatment, vol. 48, no. 2, pp. 715-726, 2016.

[96] X. Y. Shi, L. X. Xiong, L. Xiao, C. Meng, G. Y. Qi, and W. L. Li, "Downregulation of caveolin1 upregulates the expression of growth factors and regulators in coculture of fibroblasts with cancer cells," Molecular Medicine Reports, vol. 13, no. 1, pp. 744-752, 2016.

[97] A. K. Witkiewicz, A. Dasgupta, K. H. Nguyen et al., "Stromal caveolin-1 levels predict early DCIS progression to invasive 
breast cancer," Cancer Biology \& Therapy, vol. 8, no. 11, pp. 1071-1079, 2009.

[98] E. K. Sloan, D. R. Ciocca, N. Pouliot et al., "Stromal cell expression of caveolin-1 predicts outcome in breast cancer," The American Journal of Pathology, vol. 174, no. 6, pp. 2035-2043, 2009.

[99] J. S. Koo, S. Park, S. I. Kim, S. Lee, and B. W. Park, "The impact of caveolin protein expression in tumor stroma on prognosis of breast cancer," Tumour Biology, vol. 32, no. 4, pp. 787-799, 2011.

[100] N. Qian, T. Ueno, N. Kawaguchi-Sakita et al., "Prognostic significance of tumor/stromal caveolin-1 expression in breast cancer patients," Cancer Science, vol. 102, no. 8, pp. 1590-1596, 2011.

[101] S. M. El-Gendi, M. F. Mostafa, and A. M. El-Gendi, "Stromal caveolin-1 expression in breast carcinoma. Correlation with early tumor recurrence and clinical outcome," Pathology Oncology Research: POR, vol. 18, no. 2, pp. 459-469, 2012.

[102] D. Di Vizio, M. Morello, F. Sotgia, R. G. Pestell, M. R. Freeman, and M. P. Lisanti, "An absence of stromal caveolin-1 is associated with advanced prostate cancer, metastatic disease and epithelial Akt activation," Cell Cycle, vol. 8, no. 15, pp. 2420-2424, 2009.

[103] X. Zhao, Y. He, J. Gao et al., "Caveolin-1 expression level in cancer associated fibroblasts predicts outcome in gastric cancer," PloS One, vol. 8, no. 3, article e59102, 2013.

[104] L. Cantiani, M. C. Manara, C. Zucchini et al., "Caveolin-1 reduces osteosarcoma metastases by inhibiting c-Src activity and met signaling," Cancer Research, vol. 67, no. 16, pp. 7675-7685, 2007.

[105] M. Shatz and M. Liscovitch, "Caveolin-1 and cancer multidrug resistance: coordinate regulation of pro-survival proteins?” Leukemia Research, vol. 28, no. 9, pp. 907-908, 2004.

[106] A. Pang, W. Y. Au, and Y. L. Kwong, "Caveolin-1 gene is coordinately regulated with the multidrug resistance 1 gene in normal and leukemic bone marrow," Leukemia Research, vol. 28, no. 9, pp. 973-977, 2004.

[107] M. M. Belanger, M. Gaudreau, E. Roussel, and J. Couet, "Role of caveolin-1 in etoposide resistance development in A549 lung cancer cells," Cancer Biology \& Therapy, vol. 3, no. 10, pp. 954-959, 2004.

[108] C. Cai and J. Chen, "Overexpression of caveolin-1 induces alteration of multidrug resistance in Hs578T breast adenocarcinoma cells," International Journal of Cancer, vol. 111, no. 4, pp. 522-529, 2004.

[109] D. J. Stewart, "Mechanisms of resistance to cisplatin and carboplatin," Critical Reviews in Oncology/Hematology, vol. 63, no. 1, pp. 12-31, 2007.

[110] E. M. Bertino, T. M. Williams, S. P. Nana-Sinkam et al., "Stromal caveolin-1 is associated with response and survival in a phase II trial of nab-paclitaxel with carboplatin for advanced NSCLC patients," Clinical Lung Cancer, vol. 16, no. 6, pp. 466-474, 2015.

[111] G. Ayala, M. Morello, A. Frolov et al., "Loss of caveolin-1 in prostate cancer stroma correlates with reduced relapsefree survival and is functionally relevant to tumour progression," The Journal of Pathology, vol. 231, no. 1, pp. 77-87, 2013.

[112] Y. Jia, N. Wang, J. Wang et al., "Down-regulation of stromal caveolin-1 expression in esophageal squamous cell carcinoma: a potent predictor of lymph node metastases, early tumor recurrence, and poor prognosis," Annals of Surgical Oncology, vol. 21, no. 1, pp. 329-336, 2014.

[113] S. F. Yang, J. Y. Yang, C. H. Huang et al., "Increased caveolin1 expression associated with prolonged overall survival rate in hepatocellular carcinoma," Pathology, vol. 42, no. 5, pp. 438-445, 2010.

[114] Y. Ye, S. H. Miao, R. Z. Lu, and J. W. Zhou, "Prognostic value of caveolin-1 expression in gastric cancer: a meta-analysis," Asian Pacific Journal of Cancer Prevention : APJCP, vol. 15, no. 19, pp. 8367-8370, 2014.

[115] M. Shatz and M. Liscovitch, "Caveolin-1: a tumor-promoting role in human cancer," International Journal of Radiation Biology, vol. 84, no. 3, pp. 177-189, 2008.

[116] C. C. Ho, S. H. Kuo, P. H. Huang, H. Y. Huang, C. H. Yang, and P. C. Yang, "Caveolin-1 expression is significantly associated with drug resistance and poor prognosis in advanced non-small cell lung cancer patients treated with gemcitabine-based chemotherapy," Lung Cancer, vol. 59, no. 1, pp. 105-110, 2008.

[117] E. Selga, C. Morales, V. Noe, M. A. Peinado, and C. J. Ciudad, "Role of caveolin 1, E-cadherin, enolase 2 and PKCalpha on resistance to methotrexate in human HT29 colon cancer cells," BMC Medical Genomics, vol. 1, no. 1, p. 35, 2008.

[118] Z. Wang, N. Wang, W. Li et al., "Caveolin-1 mediates chemoresistance in breast cancer stem cells via beta-catenin/ ABCG2 signaling pathway," Carcinogenesis, vol. 35, no. 10, pp. 2346-2356, 2014.

[119] Z. Y. Wang, N. Wang, P. X. Liu et al., "Caveolin-1, a stressrelated oncotarget, in drug resistance," Oncotarget, vol. 6, no. 35, pp. 37135-37150, 2015.

[120] M. Tien Kuo and N. Savaraj, "Roles of reactive oxygen species in hepatocarcinogenesis and drug resistance gene expression in liver cancers," Molecular Carcinogenesis, vol. 45, no. 9, pp. 701-9, 2006.

[121] K. Kimura, S. Ito, M. Nagino, and K. Isobe, "Inhibition of reactive oxygen species down-regulates protein synthesis in RAW 264.7," Biochemical and Biophysical Research Communications, vol. 372, no. 1, pp. 272-275, 2008.

[122] A. S. Veskoukis, A. M. Tsatsakis, and D. Kouretas, "Dietary oxidative stress and antioxidant defense with an emphasis on plant extract administration," Cell Stress \& Chaperones, vol. 17, no. 1, pp. 11-21, 2012.

[123] A. Matsui, T. Ikeda, K. Enomoto et al., "Increased formation of oxidative DNA damage, 8-hydroxy-2'-deoxyguanosine, in human breast cancer tissue and its relationship to GSTP1 and COMT genotypes," Cancer Letters, vol. 151, no. 1, pp. 87-95, 2000.

[124] A. Abdul-Aziz, D. J. MacEwan, K. M. Bowles, and S. A. Rushworth, "Oxidative stress responses and NRF2 in human leukaemia," Oxidative Medicine and Cellular Longevity, vol. 2015, p. 454659, 2015.

[125] J. L. Arbiser, J. Petros, R. Klafter et al., "Reactive oxygen generated by Nox 1 triggers the angiogenic switch," Proceedings of the National Academy of Sciences of the United States of America, vol. 99, no. 2, pp. 715-720, 2002.

[126] C. Polytarchou, M. Hatziapostolou, and E. Papadimitriou, "Hydrogen peroxide stimulates proliferation and migration of human prostate cancer cells through activation of activator protein-1 and up-regulation of the heparin affin regulatory peptide gene," The Journal of Biological Chemistry, vol. 280, no. 49, pp. 40428-40435, 2005. 
[127] Y. Nakamura, T. D. Gindhart, D. Winterstein, I. Tomita, J. L. Seed, and N. H. Colburn, "Early superoxide dismutasesensitive event promotes neoplastic transformation in mouse epidermal JB6 cells," Carcinogenesis, vol. 9, no. 2, pp. 203-207, 1988.

[128] B. del Bello, A. Paolicchi, M. Comporti, A. Pompella, and E. Maellaro, "Hydrogen peroxide produced during gammaglutamyl transpeptidase activity is involved in prevention of apoptosis and maintenance of proliferation in U937 cells," FASEB Journal, vol. 13, no. 1, pp. 69-79, 1999.

[129] E. Giannoni, T. Fiaschi, G. Ramponi, and P. Chiarugi, "Redox regulation of anoikis resistance of metastatic prostate cancer cells: key role for Src and EGFR-mediated pro-survival signals," Oncogene, vol. 28, no. 20, pp. 2074-2086, 2009.

[130] U. E. Martinez-Outschoorn, C. Trimmer, Z. Lin et al., "Autophagy in cancer associated fibroblasts promotes tumor cell survival: role of hypoxia, HIF1 induction and NF kappa B activation in the tumor stromal microenvironment," Cell Cycle, vol. 9, no. 17, pp. 3515-3533, 2010.

[131] C. Trimmer, F. Sotgia, D. Whitaker-Menezes et al., "Caveolin-1 and mitochondrial SOD2 (MnSOD) function as tumor suppressors in the stromal microenvironment: a new genetically tractable model for human cancer associated fibroblasts," Cancer Biology \& Therapy, vol. 11, no. 4, pp. 383-394, 2011.

[132] A. C. Souici, J. Mirkovitch, P. Hausel, L. K. Keefer, and E. Felley-Bosco, "Transition mutation in codon 248 of the p53 tumor suppressor gene induced by reactive oxygen species and a nitric oxide-releasing compound," Carcinogenesis, vol. 21, no. 2, pp. 281-287, 2000.

[133] J. A. Coulter, H. O. McCarthy, J. Xiang et al., "Nitric oxide - a novel therapeutic for cancer," Nitric Oxide: Biology Ch, vol. 19, no. 2, pp. 192-198, 2008.

[134] L. Ying and L. J. Hofseth, "An emerging role for endothelial nitric oxide synthase in chronic inflammation and cancer," Cancer Research, vol. 67, no. 4, pp. 1407-1410, 2007.

[135] B. R. Crane, A. S. Arvai, R. Gachhui et al., "The structure of nitric oxide synthase oxygenase domain and inhibitor complexes," Science, vol. 278, no. 5337, pp. 425-431, 1997.

[136] Z. L. Chen, F. R. Bakhshi, A. N. Shajahan et al., "Nitric oxide-dependent Src activation and resultant caveolin-1 phosphorylation promote eNOS/caveolin-1 binding and eNOS inhibition," Molecular Biology of the Cell, vol. 23, no. 7, pp. 1388-1398, 2012.

[137] T. M. Williams and M. P. Lisanti, "Caveolin-1 in oncogenic transformation, cancer, and metastasis," American Journal of Physiology. Cell Physiology, vol. 288, no. 3, pp. C494-C506, 2005.

[138] N. B. Janakiram and C. V. Rao, "iNOS-selective inhibitors for cancer prevention: promise and progress," Future Medicinal Chemistry, vol. 4, no. 17, pp. 2193-2204, 2012.

[139] C. V. Rao, C. Indranie, B. Simi, P. T. Manning, J. R. Connor, and B. S. Reddy, "Chemopreventive properties of a selective inducible nitric oxide synthase inhibitor in colon carcinogenesis, administered alone or in combination with celecoxib, a selective cyclooxygenase-2 inhibitor," Cancer Research, vol. 62, no. 1, pp. 165-170, 2002.

[140] E. J. Smart, G. A. Graf, M. A. McNiven et al., "Caveolins, liquid-ordered domains, and signal transduction," Molecular and Cellular Biology, vol. 19, no. 11, pp. 7289-7304, 1999.
[141] R. Gniadecki, N. Christoffersen, and H. C. Wulf, "Cholesterol-rich plasma membrane domains (lipid rafts) in keratinocytes: importance in the baseline and UVA-induced generation of reactive oxygen species," The Journal of Investigative Dermatology, vol. 118, no. 4, pp. 582-588, 2002.

[142] A. Mougeolle, S. Poussard, M. Decossas, C. Lamaze, O. Lambert, and E. Dargelos, "Oxidative stress induces caveolin 1 degradation and impairs caveolae functions in skeletal muscle cells," PloS One, vol. 10, no. 3, article e0122654, 2015.

[143] R. P. Cai, Y. X. Xue, J. Huang et al., "NS1619 regulates the expression of caveolin-1 protein in a time-dependent manner via $\mathrm{ROS} / \mathrm{PI} 3 \mathrm{~K} / \mathrm{PKB} / \mathrm{FoxO} 1$ signaling pathway in brain tumor microvascular endothelial cells," Journal of the Neurological Sciences, vol. 369, pp. 109-118, 2016.

[144] Z. Zhang, K. Yao, and C. Jin, “Apoptosis of lens epithelial cells induced by high concentration of glucose is associated with a decrease in caveolin-1 levels," Molecular Vision, vol. 15, no. 214, pp. 2008-2017, 2009.

[145] S. Luanpitpong, S. J. Talbott, Y. Rojanasakul et al., "Regulation of lung cancer cell migration and invasion by reactive oxygen species and caveolin-1," The Journal of Biological Chemistry, vol. 285, no. 50, pp. 38832-38840, 2010.

[146] C. J. Percy, B. K. Pat, H. Healy, D. W. Johnson, and G. C. Gobe, "Phosphorylation of caveolin-1 is anti-apoptotic and promotes cell attachment during oxidative stress of kidney cells," Pathology, vol. 40, no. 7, pp. 694-701, 2008.

[147] Y. Zhang, X. Qu, Y. Teng et al., “Cbl-b inhibits P-gp transporter function by preventing its translocation into caveolae in multiple drug-resistant gastric and breast cancers," Oncotarget, vol. 6, no. 9, pp. 6737-6748, 2015.

[148] J. R. Glenney Jr., “Tyrosine phosphorylation of a $22-\mathrm{kDa}$ protein is correlated with transformation by Rous sarcoma virus," The Journal of Biological Chemistry, vol. 264, no. 34, pp. 20163-20166, 1989.

[149] C. C. Mastick, M. J. Brady, and A. R. Saltiel, "Insulin stimulates the tyrosine phosphorylation of caveolin," The Journal of Cell Biology, vol. 129, no. 6, pp. 1523-1531, 1995.

[150] M. O. Parat, R. Z. Stachowicz, and P. L. Fox, "Oxidative stress inhibits caveolin-1 palmitoylation and trafficking in endothelial cells," The Biochemical Journal, vol. 361, no. Part 3, pp. 681-688, 2002.

[151] R. Nomura and T. Fujimoto, "Tyrosine-phosphorylated caveolin-1: immunolocalization and molecular characterization," Molecular Biology of the Cell, vol. 10, no. 4, pp. 975-986, 1999.

[152] S. Wehinger, R. Ortiz, M. I. Diaz et al., "Phosphorylation of caveolin-1 on tyrosine-14 induced by ROS enhances palmitate-induced death of beta-pancreatic cells," Biochimica et Biophysica Acta, vol. 1852, no. 5, pp. 693-708, 2015.

[153] L. N. Sun, X. C. Liu, X. J. Chen, G. J. Guan, and G. Liu, "Curcumin attenuates high glucose-induced podocyte apoptosis by regulating functional connections between caveolin-1 phosphorylation and ROS," Acta Pharmacologica Sinica, vol. 37, no. 5, pp. 645-655, 2016.

[154] T. Aoki, R. Nomura, and T. Fujimoto, "Tyrosine phosphorylation of caveolin-1 in the endothelium," Experimental Cell Research, vol. 253, no. 2, pp. 629-636, 1999.

[155] A. Schlegel, P. Arvan, and M. P. Lisanti, "Caveolin-1 binding to endoplasmic reticulum membranes and entry into the regulated secretory pathway are regulated by serine 
phosphorylation protein sorting at the level of the endoplasmic reticulum," The Journal of Biological Chemistry, vol. 276, no. 6, pp. 4398-4408, 2001.

[156] X. Q. Wang, P. Sun, and A. S. Paller, "Ganglioside induces caveolin-1 redistribution and interaction with the epidermal growth factor receptor," The Journal of Biological Chemistry, vol. 277, no. 49, pp. 47028-47034, 2002.

[157] S. Blaskovic, M. Blanc, and F. G. van der Goot, "What does S-palmitoylation do to membrane proteins?" The FEBS Journal, vol. 280, no. 12, pp. 2766-2774, 2013.

[158] O. Rocks, A. Peyker, M. Kahms et al., "An acylation cycle regulates localization and activity of palmitoylated Ras isoforms," Science, vol. 307, no. 5716, pp. 1746-1752, 2005.

[159] D. J. Dietzen, W. R. Hastings, and D. M. Lublin, "Caveolin is palmitoylated on multiple cysteine residues. Palmitoylation is not necessary for localization of caveolin to caveolae," The Journal of Biological Chemistry, vol. 270, no. 12, pp. 6838-6842, 1995.

[160] A. Uittenbogaard and E. J. Smart, "Palmitoylation of caveolin-1 is required for cholesterol binding, chaperone complex formation, and rapid transport of cholesterol to caveolae," The Journal of Biological Chemistry, vol. 275, no. 33, pp. 25595-25599, 2000.

[161] H. Lee, S. E. Woodman, J. A. Engelman et al., "Palmitoylation of caveolin-1 at a single site (Cys-156) controls its coupling to the c-Src tyrosine kinase: targeting of dually acylated molecules (GPI-linked, transmembrane, or cytoplasmic) to caveolae effectively uncouples c-Src and caveolin-1 (TYR-14)," The Journal of Biological Chemistry, vol. 276, no. 37, pp. 3515035158, 2001.

[162] E. J. Smart, Y. S. Ying, P. A. Conrad, and R. G. Anderson, "Caveolin moves from caveolae to the Golgi apparatus in response to cholesterol oxidation," The Journal of Cell Biology, vol. 127, no. 5, pp. 1185-1197, 1994.

[163] A. Blair, P. W. Shaul, I. S. Yuhanna, P. A. Conrad, and E. J. Smart, "Oxidized low density lipoprotein displaces endothelial nitric-oxide synthase (eNOS) from plasmalemmal caveolae and impairs eNOS activation," The Journal of Biological Chemistry, vol. 274, no. 45, pp. 32512-32519, 1999.

[164] T. Shiroto, N. Romero, T. Sugiyama et al., "Caveolin-1 is a critical determinant of autophagy, metabolic switching, and oxidative stress in vascular endothelium," PloS One, vol. 9, no. 2, article e87871, 2014.

[165] H. Wang, A. X. Wang, Z. Liu, W. Chai, and E. J. Barrett, "The trafficking/interaction of eNOS and caveolin-1 induced by insulin modulates endothelial nitric oxide production," Molecular Endocrinology, vol. 23, no. 10, pp. 1613-1623, 2009.

[166] J. P. Gratton, M. I. Lin, J. Yu et al., "Selective inhibition of tumor microvascular permeability by cavtratin blocks tumor progression in mice," Cancer Cell, vol. 4, no. 1, pp. 31-39, 2003.

[167] H. L. Yang, W. Q. Chen, X. Cao et al., "Caveolin-1 enhances resveratrol-mediated cytotoxicity and transport in a hepatocellular carcinoma model," Journal of Translational Medicine, vol. 7, no. 1, p. 22, 2009.

[168] H. Zhan, F. Huang, W. Ma, Z. Zhao, H. Zhang, and C. Zhang, "Protective effect of ginsenoside Rg1 on bleomycin-induced pulmonary fibrosis in rats: involvement of caveolin-1 and TGF-betal signal pathway," Biological \& Pharmaceutical Bulletin, vol. 39, no. 8, pp. 1284-1292, 2016.
[169] K. Nakaso, N. Tajima, Y. Horikoshi et al., "The estrogen receptor beta-PI3K/Akt pathway mediates the cytoprotective effects of tocotrienol in a cellular Parkinson's disease model," Biochimica et Biophysica Acta, vol. 1842, no. 9, pp. 13031312, 2014.

[170] P. Palozza, R. Simone, A. Catalano, N. Parrone, G. Monego, and F. O. Ranelletti, "Lycopene regulation of cholesterol synthesis and efflux in human macrophages," The Journal of Nutritional Biochemistry, vol. 22, no. 10, pp. 971-978, 2011.

[171] M. Sanchez, M. Galisteo, R. Vera et al., "Quercetin downregulates NADPH oxidase, increases eNOS activity and prevents endothelial dysfunction in spontaneously hypertensive rats," Journal of Hypertension, vol. 24, no. 1, pp. 75-84, 2006.

[172] X. Zeng, Y. Cheng, Y. Qu, J. Xu, Z. Han, and T. Zhang, "Curcumin inhibits the proliferation of airway smooth muscle cells in vitro and in vivo," International Journal of Molecular Medicine, vol. 32, no. 3, pp. 629-636, 2013.

[173] L. N. Sun, Z. X. Chen, X. C. Liu, H. Y. Liu, G. J. Guan, and G. Liu, "Curcumin ameliorates epithelial-tomesenchymal transition of podocytes in vivo and in vitro via regulating caveolin-1," Biomedicine Pharmacotherapy Biochemical Pharmacology, vol. 68, no. 8, pp. 1079-1088, 2014.

[174] L. N. Sun, Z. Y. Yang, S. S. Lv, X. C. Liu, G. J. Guan, and G. Liu, "Curcumin prevents diabetic nephropathy against inflammatory response via reversing caveolin-1 Tyr14 phosphorylation influenced TLR4 activation," International Immunopharmacology, vol. 23, no. 1, pp. 236-246, 2014.

[175] H. Y. Yuan, S. Y. Kuang, X. Zheng et al., "Curcumin inhibits cellular cholesterol accumulation by regulating SREBP-1/caveolin-1 signaling pathway in vascular smooth muscle cells," Acta Pharmacologica Sinica, vol. 29, no. 5, pp. 555-563, 2008.

[176] O. P. Heinonen, J. K. Huttunen, D. Albanes et al., "The effect of vitamin $\mathrm{E}$ and beta carotene on the incidence of lung cancer and other cancers in male smokers. The alpha-tocopherol, beta carotene cancer prevention study group," The New England Journal of Medicine, vol. 330, no. 15, pp. 10291035, 1994.

[177] V. I. Sayin, M. X. Ibrahim, E. Larsson, J. A. Nilsson, P. Lindahl, and M. O. Bergo, "Antioxidants accelerate lung cancer progression in mice," Science Translational Medicine, vol. 6, no. 221, p. 221ra15, 2014.

[178] S. M. Lippman, P. J. Goodman, E. A. Klein et al., "Designing the Selenium and Vitamin E Cancer Prevention Trial (SELECT)," Journal of the National Cancer Institute, vol. 97, no. 2, pp. 94-102, 2005.

[179] E. A. Klein, I. M. Thompson Jr., C. M. Tangen et al., "Vitamin $\mathrm{E}$ and the risk of prostate cancer: the Selenium and Vitamin E Cancer Prevention Trial (SELECT)," Jama, vol. 306, no. 14, pp. 1549-1556, 2011.

[180] I. B. Weinstein and A. Joe, "Oncogene addiction," Cancer Research, vol. 68, no. 9, pp. 3077-3080, 2008, discussion 3080.

[181] D. R. S. Bryan and G. Allen, "Metabolic production of H2O2 in carcinogenesis and cancer treatment," in Redox-Active Therapeutics, I. B.-H. J. S. R. I. Spasojević, Ed., pp. 103-124, Springer International Publishing, Switzerland, 2016.

[182] A. L. Simons, I. M. Ahmad, D. M. Mattson, K. J. Dornfeld, and D. R. Spitz, "2-Deoxy-D-glucose combined with cisplatin enhances cytotoxicity via metabolic oxidative stress in human head and neck cancer cells," Cancer Research, vol. 67, no. 7, pp. 3364-3370, 2007. 
[183] P. C. Hart, B. A. Ratti, M. Mao et al., "Caveolin-1 regulates cancer cell metabolism via scavenging Nrf2 and suppressing MnSOD-driven glycolysis," Oncotarget, vol. 7, no. 1, pp. 308-322, 2016.

[184] F. Sotgia, U. E. Martinez-Outschoorn, S. Pavlides, A. Howell, R. G. Pestell, and M. P. Lisanti, "Understanding the Warburg effect and the prognostic value of stromal caveolin-1 as a marker of a lethal tumor microenvironment," Breast Cancer Research, vol. 13, no. 4, p. 213, 2011.

[185] G. Bonuccelli, D. Whitaker-Menezes, R. Castello-Cros et al., "The reverse Warburg effect: glycolysis inhibitors prevent the tumor promoting effects of caveolin-1 deficient cancer associated fibroblasts," Cell Cycle, vol. 9, no. 10, pp. 19601971, 2010.

[186] V. Coelho-Santos, R. Socodato, C. Portugal et al., "Methylphenidate-triggered ROS generation promotes caveolae-mediated transcytosis via Rac1 signaling and c-Src-dependent caveolin-1 phosphorylation in human brain endothelial cells," Cellular and Molecular Life Sciences: CMLS, vol. 24, no. 73, pp. 4701-4716, 2016.

[187] S. Agelaki, M. Spiliotaki, H. Markomanolaki et al., "Caveolin-1 regulates EGFR signaling in MCF-7 breast cancer cells and enhances gefitinib-induced tumor cell inhibition," Cancer Biology \& Therapy, vol. 8, no. 15, pp. 1470-1477, 2009.

[188] Z. Li, S. S. Yang, P. H. Yin et al., “Activated estrogen receptormitogen-activated protein kinases cross talk confer acquired resistance to lapatinib," Thoracic Cancer, vol. 6, no. 6, pp. 695-703, 2015.

[189] G. E. Konecny, R. Glas, J. Dering et al., "Activity of the multikinase inhibitor dasatinib against ovarian cancer cells," British Journal of Cancer, vol. 101, no. 10, pp. 1699-1708, 2009.

[190] D. Medina, F. Kittrell, J. Hill et al., "Prevention of tumorigenesis in p53-null mammary epithelium by rexinoid bexarotene, tyrosine kinase inhibitor gefitinib, and celecoxib," Cancer Prevention Research (Philadelphia, Pa.), vol. 2, no. 2, pp. 168-174, 2009.

[191] X. Wan, X. Zheng, X. Pang et al., "Lapatinib-loaded human serum albumin nanoparticles for the prevention and treatment of triple-negative breast cancer metastasis to the brain," Oncotarget, vol. 7, no. 23, pp. 3403834051, 2016.

[192] B. Gril, D. Palmieri, Y. Qian et al., "Pazopanib reveals a role for tumor cell B-Raf in the prevention of HER2+ breast cancer brain metastasis," Clinical Cancer Research: An Official Journal of the American Association for Cancer Research, vol. 17, no. 1, pp. 142-153, 2011.

[193] C. Printz, "Clinical trials of note. Sorafenib as adjuvant treatment in the prevention of disease recurrence in patients with hepatocellular carcinoma (HCC) (STORM)," Cancer, vol. 115, no. 20, p. 4646, 2009.

[194] M. P. Murphy and R. A. Smith, "Targeting antioxidants to mitochondria by conjugation to lipophilic cations," Annual Review of Pharmacology and Toxicology, vol. 47, pp. 629-656, 2007.

[195] I. W. Asterholm, D. I. Mundy, J. Weng, R. G. Anderson, and P. E. Scherer, "Altered mitochondrial function and metabolic inflexibility associated with loss of caveolin-1," Cell Metabolism, vol. 15, no. 2, pp. 171-185, 2012.
[196] M. Bosch, M. Mari, A. Herms et al., "Caveolin-1 deficiency causes cholesterol-dependent mitochondrial dysfunction and apoptotic susceptibility," Current Biology: $C B$, vol. 21, no. 8, pp. 681-686, 2011.

[197] D. Volonte, Z. Liu, S. Shiva, and F. Galbiati, "Caveolin-1 controls mitochondrial function through regulation of m-AAA mitochondrial protease," Aging, vol. 8, no. 10, pp. 2355-2369, 2016. 


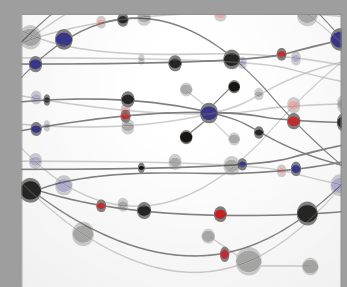

The Scientific World Journal
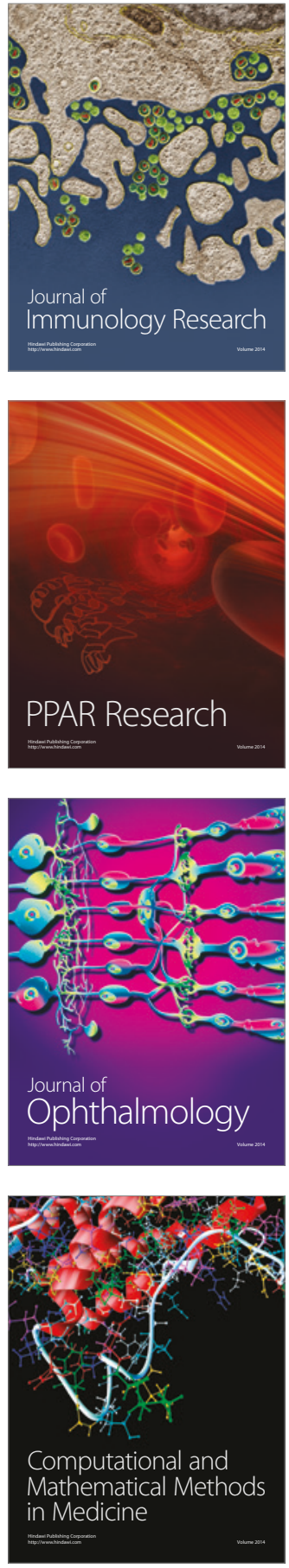

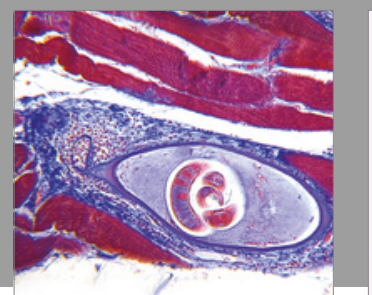

Gastroenterology Research and Practice
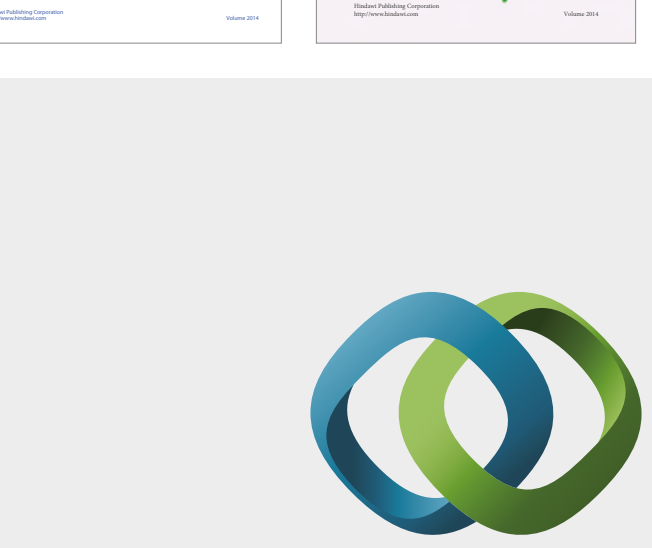

\section{Hindawi}

Submit your manuscripts at

https://www.hindawi.com
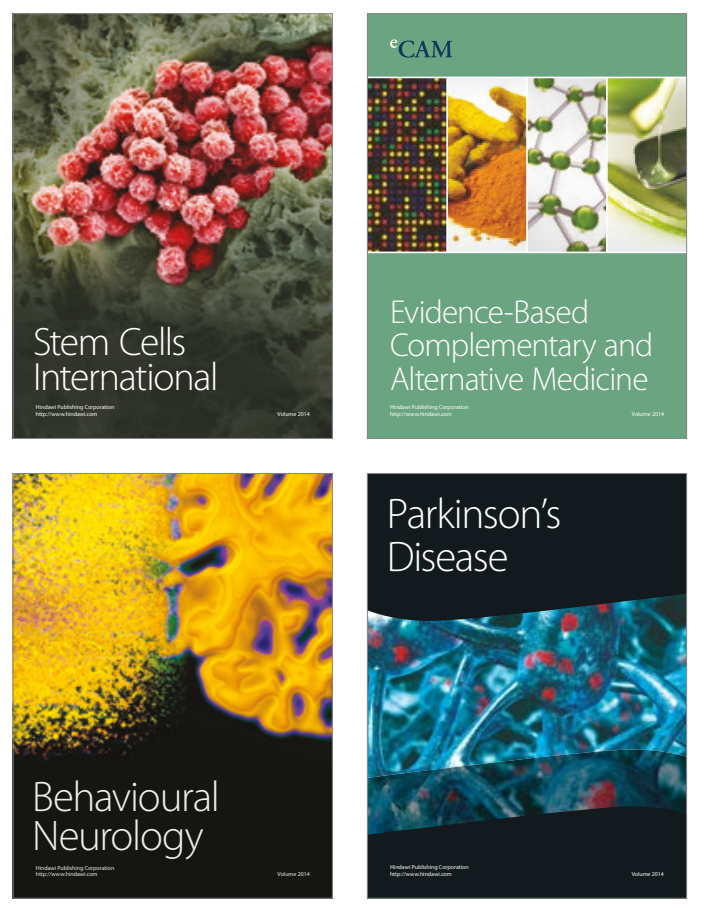
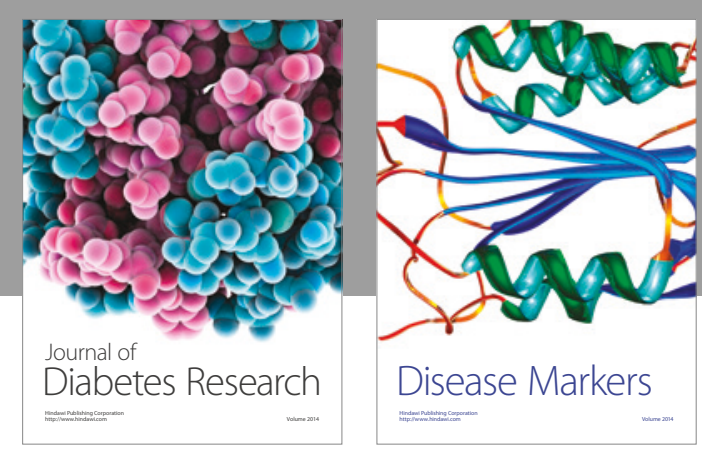

Disease Markers
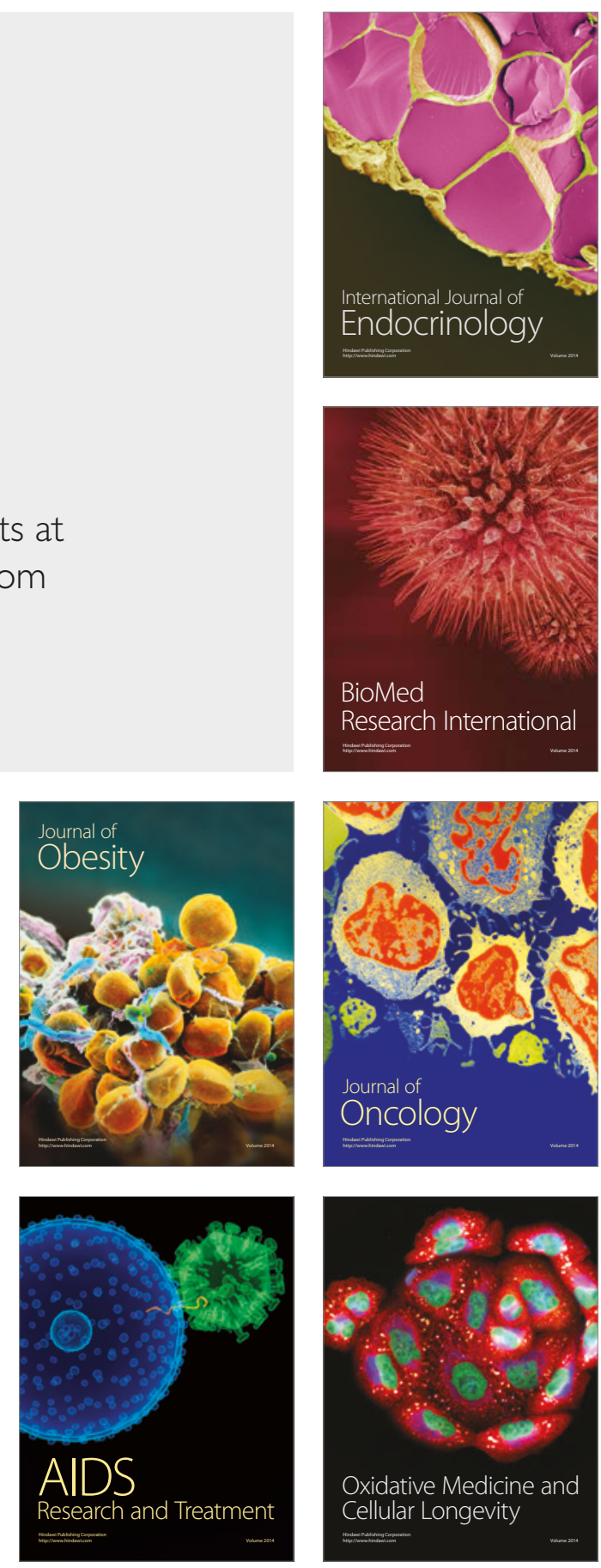